Department of Mathematics

Finite difference schemes with transfer-
able interfaces for parabolic problems

Sofia Eriksson \& Jan Nordström

LiTH-MAT-R--2018/01--SE

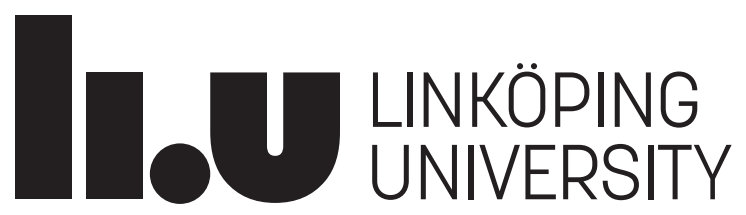


Department of Mathematics

Linköping University

S-581 83 Linköping 


\title{
Finite difference schemes with transferable interfaces for parabolic problems
}

\author{
Sofia Eriksson Jan Nordström
}

\begin{abstract}
We derive a method to locally change the order of accuracy of finite difference schemes that approximate the second derivative. The derivation is based on summation-by-parts operators, which are connected at interfaces using penalty terms. At such interfaces, the numerical solution has a double representation, with one representation in each domain. We merge this double representation into a single one, yielding a new scheme with unique solution values in all grid points. The resulting scheme is proven to be stable, accurate and dual consistent.
\end{abstract}

Keywords: Finite difference methods, summation-by-parts, high order accuracy, dual consistency, superconvergence, interfaces

\section{Introduction}

We consider summation-by-parts (SBP) finite difference methods with weakly imposed boundary and interface conditions using simultaneous approximation terms (SAT). The main advantages of the SBP-SAT technique are high accuracy, computational efficiency and provable time-stability. For a background on the SBP-SAT technique, see $[15,5]$.

Characterizing for the SAT interface treatment, is that it has free parameters that stabilize the scheme, and that the numerical solutions on both sides of the interface differ $[2,12]$. SAT interfaces are useful when generating grids for complex geometries or - as in our case - when changing the properties of the scheme in parts of the computational domain. However, if the interface must be moved, it is more convenient with one single representation of the solution in each grid point. Our approach to derive such single-valued interfaces, is to use the double-valued SAT interface treatment as a starting point, merge the double representation at the interface into a single one and obtain a new set of operators. This was done in [4] for hyperbolic problems and here we extend that methodology to parabolic problems.

The paper is organized as follows: In Section 2, we present our parabolic model problem; the heat equation with an artificial interface. This problem is discretized in space in Section 3, using a standard SAT interface. Next, in Section 4, we merge the double representation at the SAT interface into a single representation, giving us the new scheme, which is shown to be stable, accurate and dual consistent in Section 5. The theoretical results are confirmed by numerical experiments in Section 6, and a summary is given in Section 7, concluding the paper. 


\section{The model problem}

To introduce our technique we consider the one-dimensional scalar heat equation, with an interface at an interior point $\hat{x}$, as

$$
\begin{array}{ll}
u_{t}^{\mathrm{L}}=\varepsilon u_{x x}^{\mathrm{L}}, & x \in \Omega_{\mathrm{L}}=[0, \hat{x}], \\
u_{t}^{\mathrm{R}}=\varepsilon u_{x x}^{\mathrm{R}}, & x \in \Omega_{\mathrm{R}}=[\hat{x}, 1],
\end{array}
$$

which is complemented with an interface condition at $x=\hat{x}$, boundary conditions at $x=0,1$ and initial data at $t=0$.

\section{$2.1 \quad$ Well-posedness}

The problem (1) is well-posed if it has a unique solution and if it is bounded by data, see $[13,7]$. To show boundedness, we use the energy method.

We multiply the two equations in (1) by $u^{\mathrm{L}, \mathrm{R}}$ and integrate over their respective domains. Using integration by parts, and thereafter adding the two results, yields

$$
\frac{\mathrm{d}}{\mathrm{d} t}\left(\left\|u^{\mathrm{L}}\right\|^{2}+\left\|u^{\mathrm{R}}\right\|^{2}\right)+2 \varepsilon\left(\left\|u_{x}^{\mathrm{L}}\right\|^{2}+\left\|u_{x}^{\mathrm{R}}\right\|^{2}\right)=\mathrm{BT}+\mathrm{IT}
$$

where $\|u\|^{2}=\int_{0}^{1} u^{2} \mathrm{~d} x$. To make the presentation clear, we ignore the boundary terms $\mathrm{BT}=-\left.2 \varepsilon u^{\mathrm{L}} u_{x}^{\mathrm{L}}\right|_{0}+\left.2 \varepsilon u^{\mathrm{R}} u_{x}^{\mathrm{R}}\right|_{1}$ and focus on the interface terms IT $=\left.2 \varepsilon\left(u^{\mathrm{L}} u_{x}^{\mathrm{L}}-u^{\mathrm{R}} u_{x}^{\mathrm{R}}\right)\right|_{\hat{x}}$. We need IT $\leq 0$ to bound the growth of the solution. Here we aim for an artificial interface and first demand continuity, that is $\left.u^{\mathrm{L}}\right|_{\hat{x}}=\left.u^{\mathrm{R}}\right|_{\hat{x}}$. To achieve IT $=0$, we also need $\left.u_{x}^{\mathrm{L}}\right|_{\hat{x}}=\left.u_{x}^{\mathrm{R}}\right|_{\hat{x}}$. Hence, the interface conditions related to (1) are

$$
u^{\mathrm{L}}(\hat{x}, t)=u^{\mathrm{R}}(\hat{x}, t), \quad u_{x}^{\mathrm{L}}(\hat{x}, t)=u_{x}^{\mathrm{R}}(\hat{x}, t) .
$$

\section{Discretization}

We use the method of lines and discretize the spatial domain $\Omega=[0,1]$ using $N+1$ equidistant grid points $x_{j}=j h$, where $h=1 / N$ is the grid spacing and $j=0,1, \ldots, N$. Next, we introduce a numerical interface at $\hat{x}$, where $\hat{x}$ coincides with some interior grid point $x_{\hat{\imath}}$. This gives us $N_{\mathrm{L}}+1$ grid points in $\Omega_{\mathrm{L}}$ and $N_{\mathrm{R}}+1$ grid points in $\Omega_{\mathrm{R}}$, where $N_{\mathrm{L}}=\hat{\imath}$ and $N_{\mathrm{R}}=N-\hat{\imath}$.

\subsection{The SBP operators}

Let the vector $\mathbf{f}=\left[\mathbf{f}_{0}, \mathbf{f}_{1} \ldots, \mathbf{f}_{N}\right]^{\top}$ be a discrete representation of the continuous function $f(x, t)$, such that $\mathbf{f}_{j}(t)=f\left(x_{j}, t\right)$. A discrete differential operator $D_{1}$, that approximates $\partial / \partial x$ such that $\left(D_{1} \mathbf{f}\right)_{j}(t) \approx f_{x}\left(x_{j}, t\right)$, is an SBP-operator if it can be factorized as

$$
D_{1}=P^{-1} Q, \quad P=P^{\top}>0, \quad Q+Q^{\top}=E_{N}-E_{0},
$$

where $E_{0}=\mathbf{e}_{0} \mathbf{e}_{0}^{\top}, E_{N}=\mathbf{e}_{N} \mathbf{e}_{N}^{\top}, \mathbf{e}_{0}=[1,0, \ldots, 0]^{\top}$ and $\mathbf{e}_{N}=[0, \ldots, 0,1]^{\top}$. Later we use that $P$ is diagonal, and hence $D_{1}$ consists of central, $2 p$-order accurate finite difference stencils in the interior and one-sided, $p$-order accurate stencils at the boundaries $[9,10]$. 
To approximate the second derivative operator $\partial^{2} / \partial x^{2}$ we use the operator $D_{2}$, and - in addition to consistency - demand that it fulfills the SBP properties

$$
D_{2}=P^{-1}\left(-A+\mathbf{e}_{N} \mathbf{d}_{N}^{\top}-\mathbf{e}_{0} \mathbf{d}_{0}^{\top}\right), \quad A=A^{\top} \geq 0,
$$

where $\mathbf{d}_{0}^{\top} \mathbf{f} \approx f_{x}(0, t)$ and $\mathbf{d}_{N}^{\top} \mathbf{f} \approx f_{x}(1, t)$. For stability $A+A^{\top} \geq 0$ suffice, but for dual consistency we need $A$ to be symmetric [3]. The notations $>$ and $\geq$ refer to positive definite and positive semi-definite matrices, respectively, and the vectors $\mathbf{e}_{0}, \mathbf{e}_{N}, \mathbf{d}_{0}$ and $\mathbf{d}_{N}$ have dimensions $(N+1) \times 1$.

The form of $D_{2}$ in (5) is consistent with the wide-stencil case where $D_{2}^{\mathrm{w}} \equiv D_{1}^{2}$, since we, using the properties in (4), can write $D_{2}^{\mathrm{w}}=P^{-1}\left(-D_{1}^{\top} P D_{1}+\mathbf{e}_{N} \mathbf{e}_{N}^{\top} D_{1}-\mathbf{e}_{0} \mathbf{e}_{0}^{\top} D_{1}\right)$. Identifying the terms in (5), we see that in the wide-stencil case they are

$$
A^{\mathrm{w}}=D_{1}^{\top} P D_{1}, \quad \mathbf{d}_{0}^{\mathrm{w}}=D_{1}^{\top} \mathbf{e}_{0}, \quad \mathbf{d}_{N}^{\mathrm{w}}=D_{1}^{\top} \mathbf{e}_{N} .
$$

In the general case (including narrow-stencil operators) we let

$$
A=S^{\top} M S, \quad \mathbf{d}_{0}=S^{\top} \mathbf{e}_{0}, \quad \mathbf{d}_{N}=S^{\top} \mathbf{e}_{N},
$$

where the first and last row of the matrix $S$ are consistent difference stencils [2]. The interior of $S$ is not uniquely defined and neither is the matrix $M$, but they can be chosen such that $M>0$. The operators used in this work can all be found in [11].

\subsection{The SAT interface treatment}

We return to the two coupled heat equations in (1). Let $\mathbf{u}_{\mathrm{L}, \mathrm{R}}$ denote the semi-discrete approximations of $u^{\mathrm{L}, \mathrm{R}}$. We approximate $\partial^{2} / \partial x^{2}$ by $D_{2, \mathrm{~L}}$ and $D_{2, \mathrm{R}}$ in the two domains, respectively, both fulfilling the properties in (5). However, since we ignore the outer boundaries, we simplify and write

$$
D_{2, \mathrm{~L}}=P_{\mathrm{L}}^{-1}\left(-A_{\mathrm{L}}+\mathbf{e}_{N, \mathrm{~L}} \mathbf{d}_{N, \mathrm{~L}}^{\top}\right), \quad D_{2, \mathrm{R}}=P_{\mathrm{R}}^{-1}\left(-A_{\mathrm{R}}-\mathbf{e}_{0, \mathrm{R}} \mathbf{d}_{0, \mathrm{R}}^{\top}\right),
$$

for ease of presentation. The vectors $\mathbf{u}_{\mathrm{L}}, \mathbf{e}_{N, \mathrm{~L}}$ and $\mathbf{d}_{N, \mathrm{~L}}$ have dimensions $\left(N_{\mathrm{L}}+1\right) \times 1$ and the vectors $\mathbf{u}_{\mathrm{R}}, \mathbf{e}_{0, \mathrm{R}}$ and $\mathbf{d}_{0, \mathrm{R}}$ have dimensions $\left(N_{\mathrm{R}}+1\right) \times 1$, where $N_{\mathrm{L}}+N_{\mathrm{R}}=N$.

The two interface conditions from (3) are imposed using penalty terms proportional to $\mathbf{e}_{N, \mathrm{~L}}^{\top} \mathbf{u}_{\mathrm{L}}-\mathbf{e}_{0, \mathrm{R}}^{\top} \mathbf{u}_{\mathrm{R}} \approx u^{\mathrm{L}}(\hat{x}, t)-u^{\mathrm{R}}(\hat{x}, t)$ and to $\mathbf{d}_{N, \mathrm{~L}}^{\top} \mathbf{u}_{\mathrm{L}}-\mathbf{d}_{0, \mathrm{R}}^{\top} \mathbf{u}_{\mathrm{R}} \approx u_{x}^{\mathrm{L}}(\hat{x}, t)-u_{x}^{\mathrm{R}}(\hat{x}, t)$, respectively. The spatial discretization of (1) is thus given by

$$
\begin{aligned}
\frac{\mathrm{d}}{\mathrm{d} t} \mathbf{u}_{\mathrm{L}}=\varepsilon D_{2, \mathrm{~L}} \mathbf{u}_{\mathrm{L}} & +P_{\mathrm{L}}^{-1}\left(\sigma_{\mathrm{L}}^{1} \mathbf{e}_{N, \mathrm{~L}}+\sigma_{\mathrm{L}}^{2} \mathbf{d}_{N, \mathrm{~L}}\right)\left(\mathbf{e}_{N, \mathrm{~L}}^{\top} \mathbf{u}_{\mathrm{L}}-\mathbf{e}_{0, \mathrm{R}}^{\top} \mathbf{u}_{\mathrm{R}}\right) \\
& +P_{\mathrm{L}}^{-1}\left(\sigma_{\mathrm{L}}^{3} \mathbf{e}_{N, \mathrm{~L}}+\sigma_{\mathrm{L}}^{4} \mathbf{d}_{N, \mathrm{~L}}\right)\left(\mathbf{d}_{N, \mathrm{~L}}^{\top} \mathbf{u}_{\mathrm{L}}-\mathbf{d}_{0, \mathrm{R}}^{\top} \mathbf{u}_{\mathrm{R}}\right), \\
\frac{\mathrm{d}}{\mathrm{d} t} \mathbf{u}_{\mathrm{R}}=\varepsilon D_{2, \mathrm{R}} \mathbf{u}_{\mathrm{R}} & +P_{\mathrm{R}}^{-1}\left(\sigma_{\mathrm{R}}^{1} \mathbf{e}_{0, \mathrm{R}}+\sigma_{\mathrm{R}}^{2} \mathbf{d}_{0, \mathrm{R}}\right)\left(\mathbf{e}_{0, \mathrm{R}}^{\top} \mathbf{u}_{\mathrm{R}}-\mathbf{e}_{N, \mathrm{~L}}^{\top} \mathbf{u}_{\mathrm{L}}\right) \\
& +P_{\mathrm{R}}^{-1}\left(\sigma_{\mathrm{R}}^{3} \mathbf{e}_{0, \mathrm{R}}+\sigma_{\mathrm{R}}^{4} \mathbf{d}_{0, \mathrm{R}}\right)\left(\mathbf{d}_{0, \mathrm{R}}^{\top} \mathbf{u}_{\mathrm{R}}-\mathbf{d}_{N, \mathrm{~L}}^{\top} \mathbf{u}_{\mathrm{L}}\right),
\end{aligned}
$$

where the penalty parameters $\sigma_{\mathrm{L}}^{1,2,3,4}$ and $\sigma_{\mathrm{R}}^{1,2,3,4}$ will be chosen such that the scheme (8) becomes accurate, stable and dual consistent.

Remark 3.1. Choosing $\sigma_{\mathrm{L}, \mathrm{R}}^{4} \neq 0$ can decrease the accuracy, but for the sake of generality we keep these parameters in the derivations. 


\subsection{Dual consistency}

We will choose the penalty parameters $\sigma_{\mathrm{L}, \mathrm{R}}^{1,2,3,4}$ above such that the scheme becomes dual consistent [8]. We start by rewriting (8) more compactly, as

$$
\frac{\mathrm{d}}{\mathrm{d} t} \overrightarrow{\mathbf{u}}=L \overrightarrow{\mathbf{u}}
$$

where

$$
L=\varepsilon\left[\begin{array}{cc}
D_{2, \mathrm{~L}} & 0 \\
0 & D_{2, \mathrm{R}}
\end{array}\right]+\bar{P}^{-1}\left[\begin{array}{c}
\sigma_{\mathrm{L}}^{1} \mathbf{e}_{N, \mathrm{~L}}+\sigma_{\mathrm{L}}^{2} \mathbf{d}_{N, \mathrm{~L}} \\
-\sigma_{\mathrm{R}}^{1} \mathbf{e}_{0, \mathrm{R}}-\sigma_{\mathrm{R}}^{2} \mathbf{d}_{0, \mathrm{R}}
\end{array}\right] \overrightarrow{\mathbf{e}}^{\top}+\bar{P}^{-1}\left[\begin{array}{c}
\sigma_{\mathrm{L}}^{3} \mathbf{e}_{N, \mathrm{~L}}+\sigma_{\mathrm{L}}^{4} \mathbf{d}_{N, \mathrm{~L}} \\
-\sigma_{\mathrm{R}}^{3} \mathbf{e}_{0, \mathrm{R}}-\sigma_{\mathrm{R}}^{4} \mathbf{d}_{0, \mathrm{R}}
\end{array}\right] \overrightarrow{\mathbf{d}}^{\top}
$$

and

$$
\overrightarrow{\mathbf{u}}=\left[\begin{array}{c}
\mathbf{u}_{\mathrm{L}} \\
\mathbf{u}_{\mathrm{R}}
\end{array}\right], \quad \bar{P}=\left[\begin{array}{cc}
P_{\mathrm{L}} & 0 \\
0 & P_{\mathrm{R}}
\end{array}\right], \quad \overrightarrow{\mathbf{e}}=\left[\begin{array}{c}
\mathbf{e}_{N, \mathrm{~L}} \\
-\mathbf{e}_{0, \mathrm{R}}
\end{array}\right], \quad \overrightarrow{\mathbf{d}}=\left[\begin{array}{c}
\mathbf{d}_{N, \mathrm{~L}} \\
-\mathbf{d}_{0, \mathrm{R}}
\end{array}\right] .
$$

Note that the differences between the double interface representations can be expressed as $\overrightarrow{\mathbf{e}}^{\top} \overrightarrow{\mathbf{u}}=\mathbf{e}_{N, \mathrm{~L}}^{\top} \mathbf{u}_{\mathrm{L}}-\mathbf{e}_{0, \mathrm{R}}^{\top} \mathbf{u}_{\mathrm{R}}$ and $\overrightarrow{\mathbf{d}}^{\top} \overrightarrow{\mathbf{u}}=\mathbf{d}_{N, \mathrm{~L}}^{\top} \mathbf{u}_{\mathrm{L}}-\mathbf{d}_{0, \mathrm{R}}^{\top} \mathbf{u}_{\mathrm{R}}$. The vectors $\overrightarrow{\mathbf{u}}, \overrightarrow{\mathbf{e}}$ and $\overrightarrow{\mathbf{d}}$ have dimensions $(N+2) \times 1$, and $L$ and $\bar{P}$ are $(N+2) \times(N+2)$-matrices.

Now consider a continuous equation $u_{t}=\mathcal{L} u$, where $\mathcal{L}$ is a linear, spatial differential operator. Its so called dual (or adjoint) equation is $-v_{t}=\mathcal{L}^{*} v$, where the dual operator $\mathcal{L}^{*}$ is specified by $\langle v, \mathcal{L} u\rangle=\left\langle\mathcal{L}^{*} v, u\right\rangle$ and where the inner product is $\langle v, u\rangle \equiv \int_{0}^{1} v u \mathrm{~d} x$. For a scheme $\overrightarrow{\mathbf{u}}_{t}=L \overrightarrow{\mathbf{u}}$ to be a dual consistent approximation of $u_{t}=\mathcal{L} u$, we need $L^{*}$, where $L^{*}$ is the discrete dual operator, to be a consistent approximation of $\mathcal{L}^{*}$. Using the relation $\langle\overrightarrow{\mathbf{v}}, L \overrightarrow{\mathbf{u}}\rangle_{\bar{P}}=\left\langle L^{*} \overrightarrow{\mathbf{v}}, \overrightarrow{\mathbf{u}}\right\rangle_{\bar{P}}$, with $\langle\overrightarrow{\mathbf{v}}, \overrightarrow{\mathbf{u}}\rangle_{\bar{P}} \equiv \overrightarrow{\mathbf{v}}^{\top} \bar{P} \overrightarrow{\mathbf{u}}$, we find that the discrete dual operator is $L^{*} \equiv \bar{P}^{-1} L^{\top} \bar{P}[1]$.

In our case we consider $u_{t}=\varepsilon u_{x x}$, thus $\mathcal{L}=\varepsilon \partial^{2} / \partial x^{2}$. Next, we note that $\mathcal{L}$ is a selfadjoint operator, since $\mathcal{L}^{*}=\varepsilon \partial^{2} / \partial x^{2}$. This means that for (9) to be dual consistent, not only $L$ but also $L^{*}$ must be a consistent numerical approximation of $\mathcal{L}=\mathcal{L}^{*}=\varepsilon \partial^{2} / \partial x^{2}$.

We compute the discrete dual operator $L^{*}=\bar{P}^{-1} L^{\top} \bar{P}$. Using the SBP-properties (5) (here we need $A_{\mathrm{L}}, A_{\mathrm{R}}$ to be symmetric) we obtain

$$
\begin{aligned}
& L^{*}=\varepsilon\left[\begin{array}{cc}
D_{2, \mathrm{~L}} & 0 \\
0 & D_{2, \mathrm{R}}
\end{array}\right]+\bar{P}^{-1}\left[\begin{array}{cc}
\left(\sigma_{\mathrm{L}}^{1} \mathbf{e}_{N, \mathrm{~L}}+\left(\sigma_{\mathrm{L}}^{3}+\varepsilon\right) \mathbf{d}_{N, \mathrm{~L}}\right) \mathbf{e}_{N, \mathrm{~L}}^{\top} & -\left(\sigma_{\mathrm{R}}^{1} \mathbf{e}_{N, \mathrm{~L}}+\sigma_{\mathrm{R}}^{3} \mathbf{d}_{N, \mathrm{~L}}\right) \mathbf{e}_{0, \mathrm{R}}^{\top} \\
-\left(\sigma_{\mathrm{L}}^{1} \mathbf{e}_{0, \mathrm{R}}+\sigma_{\mathrm{L}}^{3} \mathbf{d}_{0, \mathrm{R}}\right) \mathbf{e}_{N, \mathrm{~L}}^{\top} & \left(\sigma_{\mathrm{R}}^{1} \mathbf{e}_{0, \mathrm{R}}+\left(\sigma_{\mathrm{R}}^{3}-\varepsilon\right) \mathbf{d}_{0, \mathrm{R}}\right) \mathbf{e}_{0, \mathrm{R}}^{\top}
\end{array}\right] \\
& +\bar{P}^{-1}\left[\begin{array}{cc}
\left(\left(\sigma_{\mathrm{L}}^{2}-\varepsilon\right) \mathbf{e}_{N, \mathrm{~L}}+\sigma_{\mathrm{L}}^{4} \mathbf{d}_{N, \mathrm{~L}}\right) \mathbf{d}_{N, \mathrm{~L}}^{\top} & -\left(\sigma_{\mathrm{R}}^{2} \mathbf{e}_{N, \mathrm{~L}}+\sigma_{\mathrm{R}}^{4} \mathbf{d}_{N, \mathrm{~L}}\right) \mathbf{d}_{0, \mathrm{R}}^{\top} \\
-\left(\sigma_{\mathrm{L}}^{2} \mathbf{e}_{0, \mathrm{R}}+\sigma_{\mathrm{L}}^{4} \mathbf{d}_{0, \mathrm{R}}\right) \mathbf{d}_{N, \mathrm{~L}}^{\top} & \left(\left(\sigma_{\mathrm{R}}^{2}+\varepsilon\right) \mathbf{e}_{0, \mathrm{R}}+\sigma_{\mathrm{R}}^{4} \mathbf{d}_{0, \mathrm{R}}\right) \mathbf{d}_{0, \mathrm{R}}^{\top}
\end{array}\right] .
\end{aligned}
$$

For $L^{*}$ to be a consistent approximation of $\mathcal{L}^{*}=\varepsilon \partial^{2} / \partial x^{2}$, it must have the same form as $L$ in (9). To be exact, the penalty portion of $L^{*}$ must consists of one part proportional to $\overrightarrow{\mathbf{e}}$ and one part proportional to $\overrightarrow{\mathbf{d}}$. This is only possible if the relations

$$
\sigma_{\mathrm{L}}^{1}=\sigma_{\mathrm{R}}^{1}, \quad \sigma_{\mathrm{L}}^{3}+\varepsilon=\sigma_{\mathrm{R}}^{3}, \quad \sigma_{\mathrm{L}}^{2}-\varepsilon=\sigma_{\mathrm{R}}^{2}, \quad \sigma_{\mathrm{L}}^{4}=\sigma_{\mathrm{R}}^{4}
$$

hold. The proportionality with $\overrightarrow{\mathbf{e}}$ and $\overrightarrow{\mathbf{d}}$ is necessary since it means that the coupling conditions $\overrightarrow{\mathbf{e}}^{\top} \overrightarrow{\mathbf{u}}=\mathbf{e}_{N, \mathrm{~L}}^{\top} \mathbf{u}_{\mathrm{L}}-\mathbf{e}_{0, \mathrm{R}}^{\top} \mathbf{u}_{\mathrm{R}} \approx 0$ and $\overrightarrow{\mathbf{d}}^{\top} \overrightarrow{\mathbf{u}}=\mathbf{d}_{N, \mathrm{~L}}^{\top} \mathbf{u}_{\mathrm{L}}-\mathbf{d}_{0, \mathrm{R}}^{\top} \mathbf{u}_{\mathrm{R}} \approx 0$ are imposed. If the penalty part of $L^{*}$ does not have this form, some other coupling conditions are imposed on the dual problem. The specific choices in (11) leads to

$$
L^{*}=\varepsilon\left[\begin{array}{cc}
D_{2, \mathrm{~L}} & 0 \\
0 & D_{2, \mathrm{R}}
\end{array}\right]+\bar{P}^{-1}\left[\begin{array}{c}
\sigma_{\mathrm{R}}^{1} \mathbf{e}_{N, \mathrm{~L}}+\sigma_{\mathrm{R}}^{3} \mathbf{d}_{N, \mathrm{~L}} \\
-\sigma_{\mathrm{L}}^{1} \mathbf{e}_{0, \mathrm{R}}-\sigma_{\mathrm{L}}^{3} \mathbf{d}_{0, \mathrm{R}}
\end{array}\right] \overrightarrow{\mathbf{e}}^{\top}+\bar{P}^{-1}\left[\begin{array}{c}
\sigma_{\mathrm{R}}^{2} \mathbf{e}_{N, \mathrm{~L}}+\sigma_{\mathrm{R}}^{4} \mathbf{d}_{N, \mathrm{~L}} \\
-\sigma_{\mathrm{L}}^{2} \mathbf{e}_{0, \mathrm{R}}-\sigma_{\mathrm{L}}^{4} \mathbf{d}_{0, \mathrm{R}}
\end{array}\right] \overrightarrow{\mathbf{d}}^{\top},
$$

which indeed is a consistent approximation of $\varepsilon \partial^{2} / \partial x^{2}$. 


\subsection{Stability}

We multiply the two equations in (8) by $\mathbf{u}_{\mathrm{L}}^{\top} P_{\mathrm{L}}$ and $\mathbf{u}_{\mathrm{R}}^{\top} P_{\mathrm{R}}$, respectively. By adding the results, we obtain the discrete analogue to (2),

$$
\begin{aligned}
\frac{\mathrm{d}}{\mathrm{d} t}\left(\mathbf{u}_{\mathrm{L}}^{\top} P_{\mathrm{L}} \mathbf{u}_{\mathrm{L}}+\mathbf{u}_{\mathrm{R}}^{\top} P_{\mathrm{R}} \mathbf{u}_{\mathrm{R}}\right)+2 \varepsilon\left(\mathbf{u}_{\mathrm{L}}^{\top} A_{\mathrm{L}} \mathbf{u}_{\mathrm{L}}+\mathbf{u}_{\mathrm{R}}^{\top} A_{\mathrm{R}} \mathbf{u}_{\mathrm{R}}\right)= \\
=\left[\begin{array}{l}
\mathbf{e}_{N, \mathrm{~L}}^{\top} \mathbf{u}_{\mathrm{L}}+\mathbf{e}_{0, \mathrm{R}}^{\top} \mathbf{u}_{\mathrm{R}} \\
\mathbf{e}_{N, \mathrm{~L}}^{\top} \mathbf{u}_{\mathrm{L}}-\mathbf{e}_{0, \mathrm{R}}^{\top} \mathbf{u}_{\mathrm{R}} \\
\mathbf{d}_{N, \mathrm{~L}}^{\top} \mathbf{u}_{\mathrm{L}}+\mathbf{d}_{0, \mathrm{R}}^{\top} \mathbf{u}_{\mathrm{R}} \\
\mathbf{d}_{N, \mathrm{~L}}^{\top} \mathbf{u}_{\mathrm{L}}-\mathbf{d}_{0, \mathrm{R}}^{\top} \mathbf{u}_{\mathrm{R}}
\end{array}\right]^{\top}\left[\begin{array}{cccc}
0 & 0 & 0 & 0 \\
0 & 2 \sigma_{\mathrm{L}}^{1} & \varepsilon & \sigma_{\mathrm{L}}^{2}+\sigma_{\mathrm{L}}^{3} \\
0 & \varepsilon & 0 & 0 \\
0 & \sigma_{\mathrm{L}}^{2}+\sigma_{\mathrm{L}}^{3} & 0 & 2 \sigma_{\mathrm{L}}^{4}
\end{array}\right]\left[\begin{array}{l}
\mathbf{e}_{N, \mathrm{~L}}^{\top} \mathbf{u}_{\mathrm{L}}+\mathbf{e}_{0, \mathrm{R}}^{\top} \mathbf{u}_{\mathrm{R}} \\
\mathbf{e}_{N, \mathrm{~L}}^{\top} \mathbf{u}_{\mathrm{L}}-\mathbf{e}_{0, \mathrm{R}}^{\top} \mathbf{u}_{\mathrm{R}} \\
\mathbf{d}_{N, \mathrm{~L}}^{\top} \mathbf{u}_{\mathrm{L}}+\mathbf{d}_{0, \mathrm{R}}^{\top} \mathbf{u}_{\mathrm{R}} \\
\mathbf{d}_{N, \mathrm{~L}}^{\top} \mathbf{u}_{\mathrm{L}}-\mathbf{d}_{0, \mathrm{R}}^{\top} \mathbf{u}_{\mathrm{R}}
\end{array}\right],
\end{aligned}
$$

where we have used the dual consistency demands (11). To show stability, the quadratic form above (containing the interface deviations) must be non-positive. However, the related matrix is indefinite for all $\varepsilon \neq 0$, regardless of the choice of penalty parameters.

To get around this, we use a variant of the technique in [3]. As indicated in (6), let $\mathbf{d}_{N, \mathrm{~L}}=S_{\mathrm{L}}^{\top} \mathbf{e}_{N, \mathrm{~L}}, \mathbf{d}_{0, \mathrm{R}}=S_{\mathrm{R}}^{\top} \mathbf{e}_{0, \mathrm{R}}$ and $A_{\mathrm{L}, \mathrm{R}}=S_{\mathrm{L}, \mathrm{R}}^{\top} M_{\mathrm{L}, \mathrm{R}} S_{\mathrm{L}, \mathrm{R}}$. We define the auxiliary variables $\mathbf{w}_{\mathrm{L}}=S_{\mathrm{L}} \mathbf{u}_{\mathrm{L}}-\frac{1}{2} M_{\mathrm{L}}^{-1} \mathbf{e}_{N, \mathrm{~L}}\left(\mathbf{e}_{N, \mathrm{~L}}^{\top} \mathbf{u}_{\mathrm{L}}-\mathbf{e}_{0, \mathrm{R}}^{\top} \mathbf{u}_{\mathrm{R}}\right)$ and $\mathbf{w}_{\mathrm{R}}=S_{\mathrm{R}} \mathbf{u}_{\mathrm{R}}-\frac{1}{2} M_{\mathrm{R}}^{-1} \mathbf{e}_{0, \mathrm{R}}\left(\mathbf{e}_{N, \mathrm{~L}}^{\top} \mathbf{u}_{\mathrm{L}}-\mathbf{e}_{0, \mathrm{R}}^{\top} \mathbf{u}_{\mathrm{R}}\right)$ and compute

$$
\begin{aligned}
& \mathbf{w}_{\mathrm{L}}^{\top} M_{\mathrm{L}} \mathbf{w}_{\mathrm{L}}=\mathbf{u}_{\mathrm{L}}^{\top} A_{L} \mathbf{u}_{\mathrm{L}}-\mathbf{d}_{N, \mathrm{~L}}^{\top} \mathbf{u}_{\mathrm{L}}\left(\mathbf{e}_{N, \mathrm{~L}}^{\top} \mathbf{u}_{\mathrm{L}}-\mathbf{e}_{0, \mathrm{R}}^{\top} \mathbf{u}_{\mathrm{R}}\right)+\frac{1}{4} q_{\mathrm{L}}\left(\mathbf{e}_{N, \mathrm{~L}}^{\top} \mathbf{u}_{\mathrm{L}}-\mathbf{e}_{0, \mathrm{R}}^{\top} \mathbf{u}_{\mathrm{R}}\right)^{2}, \\
& \mathbf{w}_{\mathrm{R}}^{\top} M_{\mathrm{R}} \mathbf{w}_{\mathrm{R}}=\mathbf{u}_{\mathrm{R}}^{\top} A_{R} \mathbf{u}_{\mathrm{R}}-\mathbf{d}_{0, \mathrm{R}}^{\top} \mathbf{u}_{\mathrm{R}}\left(\mathbf{e}_{N, \mathrm{~L}}^{\top} \mathbf{u}_{\mathrm{L}}-\mathbf{e}_{0, \mathrm{R}}^{\top} \mathbf{u}_{\mathrm{R}}\right)+\frac{1}{4} q_{\mathrm{R}}\left(\mathbf{e}_{N, \mathrm{~L}}^{\top} \mathbf{u}_{\mathrm{L}}-\mathbf{e}_{0, \mathrm{R}}^{\top} \mathbf{u}_{\mathrm{R}}\right)^{2},
\end{aligned}
$$

where $q_{\mathrm{L}}=\mathbf{e}_{N, \mathrm{~L}}^{\top} M_{\mathrm{L}}^{-1} \mathbf{e}_{N, \mathrm{~L}}$ and $q_{\mathrm{R}}=\mathbf{e}_{0, \mathrm{R}}^{\top} M_{\mathrm{R}}^{-1} \mathbf{e}_{0, \mathrm{R}}$. By replacing the terms $\mathbf{u}_{\mathrm{L}}^{\top} A_{\mathrm{L}} \mathbf{u}_{\mathrm{L}}$ and $\mathbf{u}_{\mathrm{R}}^{\top} A_{\mathrm{R}} \mathbf{u}_{\mathrm{R}}$ in (12) using the relations in (13), we obtain another discrete analogue to (2),

$$
\begin{aligned}
\frac{\mathrm{d}}{\mathrm{d} t}\left(\mathbf{u}_{\mathrm{L}}^{\top} P_{\mathrm{L}} \mathbf{u}_{\mathrm{L}}+\mathbf{u}_{\mathrm{R}}^{\top} P_{\mathrm{R}} \mathbf{u}_{\mathrm{R}}\right)+2 \varepsilon\left(\mathbf{w}_{\mathrm{L}}^{\top} M_{\mathrm{L}} \mathbf{w}_{\mathrm{L}}+\mathbf{w}_{\mathrm{R}}^{\top} M_{\mathrm{R}} \mathbf{w}_{\mathrm{R}}\right)= \\
\quad=\left[\begin{array}{c}
\mathbf{e}_{N, \mathrm{~L}}^{\top} \mathbf{u}_{\mathrm{L}}-\mathbf{e}_{0, \mathrm{R}}^{\top} \mathbf{u}_{\mathrm{R}} \\
\mathbf{d}_{N, \mathrm{~L}}^{\top} \mathbf{u}_{\mathrm{L}}-\mathbf{d}_{0, \mathrm{R}}^{\top} \mathbf{u}_{\mathrm{R}}
\end{array}\right]^{\top}\left[\begin{array}{cc}
2\left(\sigma_{\mathrm{L}}^{1}+\frac{\varepsilon}{4} q_{\mathrm{L}}+\frac{\varepsilon}{4} q_{\mathrm{R}}\right) & \sigma_{\mathrm{L}}^{2}+\sigma_{\mathrm{L}}^{3} \\
\sigma_{\mathrm{L}}^{2}+\sigma_{\mathrm{L}}^{3} & 2 \sigma_{\mathrm{L}}^{4}
\end{array}\right]\left[\begin{array}{c}
\mathbf{e}_{N, \mathrm{~L}}^{\top} \mathbf{u}_{\mathrm{L}}-\mathbf{e}_{0, \mathrm{R}}^{\top} \mathbf{u}_{\mathrm{R}} \\
\mathbf{d}_{N, \mathrm{~L}}^{\top} \mathbf{u}_{\mathrm{L}}-\mathbf{d}_{0, \mathrm{R}}^{\top} \mathbf{u}_{\mathrm{R}}
\end{array}\right] .
\end{aligned}
$$

Our particular choice of auxiliary variables $\mathbf{w}_{\mathrm{L}}$ and $\mathbf{w}_{\mathrm{R}}$ has removed the problematic mixed terms in (12). The scheme will be stable (and dual consistent) for all sets of interface penalty parameters having the form

$$
\sigma_{\mathrm{L}}^{1}=\sigma_{\mathrm{R}}^{1}=s_{1}-\varepsilon \frac{q_{\mathrm{L}}+q_{\mathrm{R}}}{4}, \quad \begin{array}{ll}
\sigma_{\mathrm{L}}^{2}=s_{2}+\varepsilon / 2 \\
\sigma_{\mathrm{R}}^{2}=s_{2}-\varepsilon / 2
\end{array}, \quad \begin{aligned}
& \sigma_{\mathrm{L}}^{3}=s_{3}-\varepsilon / 2 \\
& \sigma_{\mathrm{R}}^{3}=s_{3}+\varepsilon / 2
\end{aligned}, \quad \sigma_{\mathrm{L}}^{4}=\sigma_{\mathrm{R}}^{4}=s_{4},
$$

where the parameters $s_{1,2,3,4}$ must fulfill $s_{1} \leq 0, s_{4} \leq 0$ and $\left(s_{2}+s_{3}\right)^{2} \leq 4 s_{1} s_{4}$.

Remark 3.2. Just as $S_{\mathrm{L}, \mathrm{R}} \mathbf{u}_{\mathrm{L}, \mathrm{R}}$, the auxiliary variables $\mathbf{w}_{\mathrm{L}, \mathrm{R}}$, which have been modified with penalty-like terms, are consistent approximations of $u_{x}$ in the first and last row.

Remark 3.3. For narrow-stencil second derivative operators, the stability demands on the penalty parameters actually depend on both boundaries. Here we have neglected the outer boundaries when defining the auxiliary variables $\mathbf{w}_{\mathrm{L}, \mathrm{R}}$, but if they are included in the derivation, it affects $q_{\mathrm{L}, \mathrm{R}}$ slightly. This modification (insignificant for fine grids), is accounted for in the $q$-values tabulated in [3]. 


\section{Transformation into a single-valued interface}

Equipped with a stable and dual consistent multi-domain formulation, we now turn to our main task. Using (7) and (14), the scheme (9) can be written

$$
\begin{aligned}
\frac{\mathrm{d}}{\mathrm{d} t} \overrightarrow{\mathbf{u}}=-\varepsilon \bar{P}^{-1}\left[\begin{array}{cc}
A_{\mathrm{L}} & 0 \\
0 & A_{\mathrm{R}}
\end{array}\right] \overrightarrow{\mathbf{u}} & +\sigma_{\mathrm{L}, \mathrm{R}}^{1} \bar{P}^{-1} \overrightarrow{\mathbf{e}}^{\top} \overrightarrow{\mathbf{u}}+\bar{P}^{-1}\left[\begin{array}{c}
\left(\varepsilon / 2+s_{2}\right) \mathbf{d}_{N, \mathrm{~L}} \\
\left(\varepsilon / 2-s_{2}\right) \mathbf{d}_{0, \mathrm{R}}
\end{array}\right] \overrightarrow{\mathbf{e}}^{\top} \overrightarrow{\mathbf{u}} \\
& +\bar{P}^{-1} \overrightarrow{\mathbf{e}}\left[\begin{array}{c}
\left(\varepsilon / 2+s_{3}\right) \mathbf{d}_{N, \mathrm{~L}} \\
\left(\varepsilon / 2-s_{3}\right) \mathbf{d}_{0, \mathrm{R}}
\end{array}\right]^{\top} \overrightarrow{\mathbf{u}}+s_{4} \bar{P}^{-1} \overrightarrow{\mathbf{d}}^{\top} \overrightarrow{\mathbf{u}}
\end{aligned}
$$

Note that both $\mathbf{e}_{N, \mathrm{~L}}^{\top} \mathbf{u}_{\mathrm{L}}$ and $\mathbf{e}_{0, \mathrm{R}}^{\top} \mathbf{u}_{\mathrm{R}}$ in $\overrightarrow{\mathbf{e}}^{\top} \overrightarrow{\mathbf{u}}=\mathbf{e}_{N, \mathrm{~L}}^{\top} \mathbf{u}_{\mathrm{L}}-\mathbf{e}_{0, \mathrm{R}}^{\top} \mathbf{u}_{\mathrm{R}} \approx 0$ are approximations of the exact solution value $u(\hat{x}, t)$. Our aim is to modify the scheme such that it operates without this double representation at the interface.

\subsection{Derivation of the new scheme}

We start by defining the matrices $\widetilde{K}$ and $\widetilde{I}$. Their respective purpose is to merge and to duplicate interface values. They are similar to the identity matrix, but their dimensions are $(N+1) \times(N+2)$ and they are modified in the interior: Around row $\hat{\imath}$ and columns $\hat{\imath}$ and $\hat{\imath}+1$ they have the entries

$$
\widetilde{K}=\left[\begin{array}{cccccc}
\ddots & & & & & \\
& 1 & 0 & 0 & 0 & \\
& 0 & \alpha & 1-\alpha & 0 & \\
& 0 & 0 & 1 & \\
& & & & & \ddots
\end{array}\right], \quad \widetilde{I}=\left[\begin{array}{llllll}
\ddots & & & & & \\
& 1 & 0 & 0 & 0 & \\
& 0 & 1 & 1 & 0 & \\
& 0 & 0 & 0 & 1 & \\
& & & & & \ddots
\end{array}\right]
$$

where $\alpha=\mathbf{e}_{N, \mathrm{~L}}^{\top} P_{\mathrm{L}} \mathbf{e}_{N, \mathrm{~L}} /\left(\mathbf{e}_{N, \mathrm{~L}}^{\top} P_{\mathrm{L}} \mathbf{e}_{N, \mathrm{~L}}+\mathbf{e}_{0, \mathrm{R}}^{\top} P_{\mathrm{R}} \mathbf{e}_{0, \mathrm{R}}\right)$. For later reference, we note that

$$
\widetilde{I}\left[\begin{array}{c}
c_{\mathrm{L}} \mathbf{e}_{N, \mathrm{~L}} \\
c_{\mathrm{R}} \mathbf{e}_{0, \mathrm{R}}
\end{array}\right]=\left(c_{\mathrm{L}}+c_{\mathrm{R}}\right) \mathbf{e}_{\hat{\imath}}, \quad \mathbf{e}_{\hat{\imath}}^{\top} \widetilde{K}=\left[\begin{array}{c}
\alpha \mathbf{e}_{N, \mathrm{~L}} \\
(1-\alpha) \mathbf{e}_{0, \mathrm{R}}
\end{array}\right]^{\top}, \quad \mathbf{e}_{\hat{\imath}}=[0, \ldots, 0,1,0, \ldots, 0]^{\top},
$$

where $c_{\mathrm{L}, \mathrm{R}}$ are arbitrary scalars and $\mathbf{e}_{\hat{\imath}}$ is a $(N+1) \times 1$-vector, non-zero only in row $\hat{\imath}$. In particular, note that $\widetilde{I} \overrightarrow{\mathbf{e}}=(1-1) \mathbf{e}_{\hat{\imath}}=0$, where $\overrightarrow{\mathbf{e}}=[0, \ldots, 0,1,-1,0, \ldots, 0]^{\top}$ is given in $(10)$.

Proposition 4.1. Consider the diagonal matrix $\bar{P}$ in (10). With $\widetilde{K}$ and $\widetilde{I}$ as specified in (16), the relation $\widetilde{K} \bar{P}^{-1}=\widetilde{P}^{-1} \widetilde{I}$ holds, where $\widetilde{P}$ is defined as $\widetilde{P} \equiv \widetilde{I} \bar{P} \widetilde{I}^{\top}$.

Assumption 4.2. $\mathbf{e}_{N, L}^{\top} \mathbf{u}_{L} \equiv \mathbf{e}_{0, R}^{\top} \mathbf{u}_{R}$.

Corollary 4.3. Assumption 4.2 leads to the relation $\overrightarrow{\mathbf{u}}=\widetilde{I}^{\top} \widetilde{K} \overrightarrow{\mathbf{u}}$, where $\overrightarrow{\mathbf{u}}$ is given in (10) and $\widetilde{K}$ and $\widetilde{I}$ are given in (16).

Corollary 4.3 and Proposition 4.1 are proven in [4]. For completeness we provide the proofs in Appendix A. 
We are now ready to derive the new scheme: Since we aim for a single solution value at the interface instead of two, we multiply our original scheme (15) by $\widetilde{K}$ from the left. We thereafter use $\widetilde{K} \bar{P}^{-1}=\widetilde{P}^{-1} \widetilde{I}$ from Proposition 4.1, yielding

$$
\begin{aligned}
\widetilde{K} \frac{\mathrm{d}}{\mathrm{d} t} \overrightarrow{\mathbf{u}} & =-\varepsilon \widetilde{P}^{-1} \widetilde{I}\left[\begin{array}{cc}
A_{\mathrm{L}} & 0 \\
0 & A_{\mathrm{R}}
\end{array}\right] \overrightarrow{\mathbf{u}}+\sigma_{\mathrm{L}, \mathrm{R}}^{1} \widetilde{P}^{-1} \widetilde{I}_{\overrightarrow{\mathbf{e}}^{\top}} \overrightarrow{\mathbf{u}}^{\top}+\widetilde{P}^{-1} \widetilde{I}\left[\begin{array}{c}
\left(\varepsilon / 2+s_{2}\right) \mathbf{d}_{N, \mathrm{~L}} \\
\left(\varepsilon / 2-s_{2}\right) \mathbf{d}_{0, \mathrm{R}}
\end{array}\right] \overrightarrow{\mathbf{e}}^{\top} \overrightarrow{\mathbf{u}} \\
& +\widetilde{P}^{-1} \widetilde{I} \overrightarrow{\mathbf{e}}\left[\begin{array}{c}
\left(\varepsilon / 2+s_{3}\right) \mathbf{d}_{N, \mathrm{~L}} \\
\left(\varepsilon / 2-s_{3}\right) \mathbf{d}_{0, \mathrm{R}}
\end{array}\right]^{\top} \overrightarrow{\mathbf{u}}+s_{4} \widetilde{P}^{-1} \widetilde{I} \overrightarrow{\mathbf{d}}^{\top} \overrightarrow{\mathbf{d}}^{\top} \overrightarrow{\mathbf{u}} .
\end{aligned}
$$

Let $\widetilde{K}$ be constant, such that $\widetilde{K} \overrightarrow{\mathbf{u}}_{t}=(\widetilde{K} \overrightarrow{\mathbf{u}})_{t}$ and define $\widetilde{\mathbf{u}} \equiv \widetilde{K} \overrightarrow{\mathbf{u}}$. The vector $\widetilde{\mathbf{u}}$ is one element shorter than $\overrightarrow{\mathbf{u}}$ and identical to $\overrightarrow{\mathbf{u}}$ in all points, except at the interface, where $\mathbf{e}_{\hat{\imath}}^{\top} \widetilde{\mathbf{u}}=\alpha \mathbf{e}_{N, \mathrm{~L}}^{\top} \mathbf{u}_{\mathrm{L}}+(1-\alpha) \mathbf{e}_{0, \mathrm{R}}^{\top} \mathbf{u}_{\mathrm{R}}$.

Next, Assumption 4.2 yields $\overrightarrow{\mathbf{e}}^{\top} \overrightarrow{\mathbf{u}}=\mathbf{e}_{N, \mathrm{~L}}^{\top} \mathbf{u}_{\mathrm{L}}-\mathbf{e}_{0, \mathrm{R}}^{\top} \mathbf{u}_{\mathrm{R}}=0$, which removes the first two penalty terms above. The relation $\widetilde{I} \overrightarrow{\mathbf{e}}=0$ from (17) removes the third penalty term. We proceed by replacing $\widetilde{K} \overrightarrow{\mathbf{u}}$ by $\widetilde{\mathbf{u}}$ and thereafter, using Corollary 4.3, every remaining $\overrightarrow{\mathbf{u}}$ by $\widetilde{I}^{\top} \widetilde{K} \overrightarrow{\mathbf{u}}=\widetilde{I}^{\top} \widetilde{\mathbf{u}}$. These steps yield

$$
\frac{\mathrm{d}}{\mathrm{d} t} \widetilde{\mathbf{u}}=-\varepsilon \widetilde{P}^{-1} \widetilde{I}\left[\begin{array}{cc}
A_{\mathrm{L}} & 0 \\
0 & A_{\mathrm{R}}
\end{array}\right] \widetilde{I}^{\top} \widetilde{\mathbf{u}}+s_{4} \widetilde{P}^{-1} \widetilde{I} \overrightarrow{\mathbf{d}} \overrightarrow{\mathbf{d}}^{\top} \widetilde{I}^{\top} \widetilde{\mathbf{u}}
$$

with $s_{4} \leq 0$ as an optional damping parameter.

If the second order accurate narrow-stencil operator is used in both domains, $s_{4}=0$ will result in the second order stencil in the whole domain, without any special features at the interface. Moreover, numerical experiments suggests that $s_{4} \neq 0$ gives higher cancellation errors. This, in addition to simplicity, speaks in favor for $s_{4}=0$. With that choice, our final scheme is

$$
\frac{\mathrm{d}}{\mathrm{d} t} \widetilde{\mathbf{u}}=-\varepsilon \widetilde{P}^{-1} \widetilde{A} \widetilde{\mathbf{u}}, \quad \widetilde{P}=\widetilde{I}\left[\begin{array}{cc}
P_{\mathrm{L}} & 0 \\
0 & P_{\mathrm{R}}
\end{array}\right] \widetilde{I}^{\top}, \quad \widetilde{A}=\widetilde{I}\left[\begin{array}{cc}
A_{\mathrm{L}} & 0 \\
0 & A_{\mathrm{R}}
\end{array}\right] \widetilde{I}^{\top}
$$

This concludes the derivation. At this point we can forget Assumption 4.2, since (18) is a new scheme, independent of the original one.

\section{Properties of the new scheme}

In the derivation of the new scheme (18), we rather boldly required that the original solution vector $\overrightarrow{\mathbf{u}}$ fulfilled Assumption 4.2, and initially the new solution vector $\widetilde{\mathbf{u}}$ was related to $\overrightarrow{\mathbf{u}}$. Below we show that our final scheme (18) is in fact a stand-alone scheme, with the SBP-properties preserved.

\subsection{Stability}

First, it is easily seen that the scheme is stable, since $\widetilde{P}>0$ and $\widetilde{A} \geq 0$. Multiplying (18) from the left by $\widetilde{\mathbf{u}}^{\top} \widetilde{P}$, we directly obtain the energy decay

$$
\frac{\mathrm{d}}{\mathrm{d} t}\left(\widetilde{\mathbf{u}}^{\top} \widetilde{P} \widetilde{\mathbf{u}}\right)+2 \varepsilon \widetilde{\mathbf{u}}^{\top} \widetilde{A} \widetilde{\mathbf{u}}=0
$$


Exploiting that $\widetilde{I}^{\top} \widetilde{\mathbf{u}}=\left[\widetilde{\mathbf{u}}_{\mathrm{L}}^{\top}, \widetilde{\mathbf{u}}_{\mathrm{R}}^{\top}\right]^{\top}$, where $\widetilde{\mathbf{u}}_{\mathrm{L}}$ refers to the left part of $\widetilde{\mathbf{u}}$ (including the interface value $\mathbf{e}_{\hat{\imath}}^{\top} \widetilde{\mathbf{u}}$ ) and $\widetilde{\mathbf{u}}_{\mathrm{R}}$ refers to the right part of $\widetilde{\mathbf{u}}$ (also including $\mathbf{e}_{\hat{\imath}}^{\top} \widetilde{\mathbf{u}}$ ), we can rewrite the above growth rate in an equivalent form, as

$$
\frac{\mathrm{d}}{\mathrm{d} t}\left(\widetilde{\mathbf{u}}_{\mathrm{L}}^{\top} P_{\mathrm{L}} \widetilde{\mathbf{u}}_{\mathrm{L}}+\widetilde{\mathbf{u}}_{\mathrm{R}}^{\top} P_{\mathrm{R}} \widetilde{\mathbf{u}}_{\mathrm{R}}\right)+2 \varepsilon\left(\widetilde{\mathbf{u}}_{\mathrm{L}}^{\top} A_{\mathrm{L}} \widetilde{\mathbf{u}}_{\mathrm{L}}+\widetilde{\mathbf{u}}_{\mathrm{R}}^{\top} A_{\mathrm{R}} \widetilde{\mathbf{u}}_{\mathrm{R}}\right)=0
$$

which more clearly resembles (2). Recall that we omit the contribution from the outer boundaries.

\subsection{Accuracy}

Next, we show that the new scheme is accurate. Using (5), Proposition 4.1 and (17), the scheme (18) can be rewritten as

$$
\frac{\mathrm{d}}{\mathrm{d} t} \widetilde{\mathbf{u}}=\varepsilon \widetilde{K}\left[\begin{array}{c}
D_{2, \mathrm{~L}} \widetilde{\mathbf{u}}_{\mathrm{L}} \\
D_{2, \mathrm{R}} \widetilde{\mathbf{u}}_{\mathrm{R}}
\end{array}\right]-\varepsilon \widetilde{P}^{-1} \mathbf{e}_{\hat{\imath}}\left(\mathbf{d}_{N, \mathrm{~L}}^{\top} \widetilde{\mathbf{u}}_{\mathrm{L}}-\mathbf{d}_{0, \mathrm{R}}^{\top} \widetilde{\mathbf{u}}_{\mathrm{R}}\right)
$$

Evaluating (19) point-wise, we obtain

$$
\frac{\mathrm{d}}{\mathrm{d} t}\left(\mathbf{e}_{\hat{\imath}}^{\top} \widetilde{\mathbf{u}}\right)=\varepsilon\left(\alpha\left(D_{2, \mathrm{~L}} \widetilde{\mathbf{u}}_{\mathrm{L}}\right)_{N_{\mathrm{L}}}+(1-\alpha)\left(D_{2, \mathrm{R}} \widetilde{\mathbf{u}}_{\mathrm{R}}\right)_{0}\right)-\varepsilon \mathbf{e}_{\hat{\imath}}^{\top} \widetilde{P}^{-1} \mathbf{e}_{\hat{\imath}}\left(\mathbf{d}_{N, \mathrm{~L}}^{\top} \widetilde{\mathbf{u}}_{\mathrm{L}}-\mathbf{d}_{0, \mathrm{R}}^{\top} \widetilde{\mathbf{u}}_{\mathrm{R}}\right)
$$

at the interface and

$$
\frac{\mathrm{d}}{\mathrm{d} t}\left(\mathbf{e}_{j}^{\top} \widetilde{\mathbf{u}}\right)= \begin{cases}\varepsilon\left(D_{2, \mathrm{~L}} \widetilde{\mathbf{u}}_{\mathrm{L}}\right)_{j}, & 0 \leq j<\hat{\imath} \\ \varepsilon\left(D_{2, \mathrm{R}} \widetilde{\mathbf{u}}_{\mathrm{R}}\right)_{j-\hat{\imath}}, & \hat{\imath}<j \leq N\end{cases}
$$

elsewhere. At the interface, the scheme is hence nothing but two one-sided operators weighted together, plus an in-built internal penalty on the second interface condition.

Let $\underline{u}, \underline{u}_{\mathrm{L}}$ and $\underline{u}_{\mathrm{R}}$ denote the exact solution (projected on to the grids in $\Omega, \Omega_{\mathrm{L}}$ and $\Omega_{\mathrm{R}}$, respectively). Note that $\mathbf{e}_{N, \mathrm{~L}}^{\top} \underline{u}_{\mathrm{L}}=\mathbf{e}_{\hat{\imath}}^{\top} \underline{u}=\mathbf{e}_{0, \mathrm{R}}^{\top} \underline{u}_{\mathrm{R}}$. Inserting $\underline{u}_{\mathrm{L}, \mathrm{R}}$ into (8) yields the truncation errors

$$
\begin{aligned}
T_{e}^{\mathrm{L}} & =\frac{\mathrm{d}}{\mathrm{d} t} \underline{u}_{\mathrm{L}}-\varepsilon D_{2, \mathrm{~L}} \underline{u}_{\mathrm{L}}-P_{\mathrm{L}}^{-1}\left(\sigma_{\mathrm{L}}^{3} \mathbf{e}_{N, \mathrm{~L}}+\sigma_{\mathrm{L}}^{4} \mathbf{d}_{N, \mathrm{~L}}\right)\left(\mathbf{d}_{N, \mathrm{~L}}^{\top} \underline{u}_{\mathrm{L}}-\mathbf{d}_{0, \mathrm{R}}^{\top} \underline{u}_{\mathrm{R}}\right), \\
T_{e}^{\mathrm{R}} & =\frac{\mathrm{d}}{\mathrm{d} t} \underline{u}_{\mathrm{R}}-\varepsilon D_{2, \mathrm{R}} \underline{u}_{\mathrm{R}}-P_{\mathrm{R}}^{-1}\left(\sigma_{\mathrm{R}}^{3} \mathbf{e}_{0, \mathrm{R}}+\sigma_{\mathrm{R}}^{4} \mathbf{d}_{0, \mathrm{R}}\right)\left(\mathbf{d}_{0, \mathrm{R}}^{\top} \underline{u}_{\mathrm{R}}-\mathbf{d}_{N, \mathrm{~L}}^{\top} \underline{u}_{\mathrm{L}}\right),
\end{aligned}
$$

and inserting $\underline{u}$ into the new scheme (19) produces the truncation error

$$
\widetilde{T}_{e}=\frac{\mathrm{d}}{\mathrm{d} t} \underline{u}-\varepsilon \widetilde{K}\left[\begin{array}{c}
D_{2, \mathrm{~L}} \underline{u}_{\mathrm{L}} \\
D_{2, \mathrm{R}} \underline{u}_{\mathrm{R}}
\end{array}\right]+\varepsilon \widetilde{P}^{-1} \mathbf{e}_{\hat{\imath}}\left(\mathbf{d}_{N, \mathrm{~L}}^{\top} \underline{u}_{\mathrm{L}}-\mathbf{d}_{0, \mathrm{R}}^{\top} \underline{u}_{\mathrm{R}}\right) .
$$

With the penalty parameters in (8) chosen according to (14), it can be shown that

$$
\widetilde{T}_{e}=\widetilde{K}\left[\begin{array}{l}
T_{e}^{\mathrm{L}} \\
T_{e}^{\mathrm{R}}
\end{array}\right], \quad \mathbf{e}_{j}^{\mathrm{T}} \widetilde{T}_{e}=\left\{\begin{array}{lc}
\left(T_{e}^{\mathrm{L}}\right)_{j} & 0 \leq j<\hat{\imath} \\
\alpha\left(T_{e}^{\mathrm{L}}\right)_{N_{\mathrm{L}}}+(1-\alpha)\left(T_{e}^{\mathrm{R}}\right)_{0}, & j=\hat{\imath} \\
\left(T_{e}^{\mathrm{R}}\right)_{j-\hat{\imath}} & \hat{\imath}<j \leq N .
\end{array}\right.
$$

The truncation errors from the original SAT interface scheme and from the new scheme are thus identical, except at the interface. Just as for the original scheme, the global order of accuracy of the new scheme is the same as that of the operator $D_{2, \mathrm{~L}}$ or $D_{2, \mathrm{R}}$ which has the lowest order. 


\subsection{Dual consistency}

Finally, let $\widetilde{L}=-\varepsilon \widetilde{P}^{-1} \widetilde{A}$ denote the linear, spatial operator in (18). Computing its dual operator as $\widetilde{L}^{*}=\widetilde{P}^{-1} \widetilde{L}^{\top} \widetilde{P}$, we obtain $\widetilde{L}^{*}=-\varepsilon \widetilde{P}^{-1} \widetilde{A}^{\top}=\widetilde{L}$, where the last equality holds since $\widetilde{A}$ is symmetric. Thus $\widetilde{L}$ is self-adjoint and the scheme (18) is dual consistent (given that the outer boundary conditions are imposed in a dual consistent manner).

\section{Numerical simulations}

Consider the advection-diffusion equation with Dirichlet boundary conditions, that is

$$
\begin{aligned}
u_{t}+a u_{x} & =\varepsilon u_{x x}+f, & & x \in(0,1), \\
u & =g_{\mathrm{L}}, & & x=0, \\
u & =g_{\mathrm{R}}, & & x=1,
\end{aligned}
$$

where $f, g_{\mathrm{L}}$ and $g_{\mathrm{R}}$ are given data. We want to solve (20) using the new scheme

$$
\begin{aligned}
\mathbf{u}_{t}+a \widetilde{P}^{-1} \widetilde{Q} \mathbf{u} & =\varepsilon \widetilde{P}^{-1}\left(-\widetilde{A}+\mathbf{e}_{N} \widetilde{\mathbf{d}}_{N}^{\top}-\mathbf{e}_{0} \widetilde{\mathbf{d}}_{0}^{\top}\right) \mathbf{u}+\mathbf{f} \\
& +\widetilde{P}^{-1}\left(\mu_{0} \mathbf{e}_{0}+\nu_{0} \widetilde{\mathbf{d}}_{0}\right)\left(\mathbf{e}_{0}^{\top} \mathbf{u}-g_{\mathrm{L}}\right) \\
& +\widetilde{P}^{-1}\left(\mu_{N} \mathbf{e}_{N}+\nu_{N} \widetilde{\mathbf{d}}_{N}\right)\left(\mathbf{e}_{N}^{\top} \mathbf{u}-g_{\mathrm{R}}\right),
\end{aligned}
$$

where $\mathbf{f}$ is the restriction of $f$ to the grid. $\widetilde{P}$ and $\widetilde{A}$ are given in (18), and the difference matrix $\widetilde{Q}=\widetilde{I} \bar{Q} \widetilde{I}^{\top}$, with $\bar{Q}$ given below, was derived in [4]. Moreover, since we now include the outer boundaries, we need $\widetilde{\mathbf{d}}_{0}=\widetilde{I} \bar{S}^{\top} \widetilde{I}^{\top} \mathbf{e}_{0}$ and $\widetilde{\mathbf{d}_{N}}=\widetilde{I} \bar{S}^{\top} \widetilde{I}^{\top} \mathbf{e}_{N}$, with $\bar{S}$ given below (together with $\bar{M}$ which is needed for the penalty parameters). We have

$$
\bar{Q}=\left[\begin{array}{cc}
Q_{\mathrm{L}} & 0 \\
0 & Q_{\mathrm{R}}
\end{array}\right], \quad \bar{S}=\left[\begin{array}{cc}
S_{\mathrm{L}} & 0 \\
0 & S_{\mathrm{R}}
\end{array}\right], \quad \bar{M}=\left[\begin{array}{cc}
M_{\mathrm{L}} & 0 \\
0 & M_{\mathrm{R}}
\end{array}\right],
$$

where $Q_{\mathrm{L}, \mathrm{R}}$ are both fulfilling (4) and where $S_{\mathrm{L}, \mathrm{R}}$ and $M_{\mathrm{L}, \mathrm{R}}$ are associated with $A_{\mathrm{L}, \mathrm{R}}$ in $\widetilde{A}$, as specified in (5) and (6). In (21), we use the penalty parameters

$$
\begin{array}{rlrl}
\mu_{0} & =-\frac{a+\omega_{\mathrm{L}}}{2}-q_{\mathrm{L}} \varepsilon, & \nu_{0}=-\varepsilon, & \omega_{\mathrm{L}}>0, \\
\mu_{N}=\frac{a-\omega_{\mathrm{R}}}{2}-q_{\mathrm{R}} \varepsilon, & \nu_{N}=\varepsilon, & \omega_{\mathrm{R}}>0,
\end{array}
$$

from [3], which are designed to give a stable and dual consistent numerical solution. For $q_{\mathrm{L}}=\mathbf{e}_{0}^{\top} \widetilde{I} \bar{M}^{-1} \widetilde{I}^{\top} \mathbf{e}_{0}=\mathbf{e}_{0, \mathrm{~L}}^{\top} M_{\mathrm{L}}^{-1} \mathbf{e}_{0, \mathrm{~L}}$ and $q_{\mathrm{R}}=\mathbf{e}_{N}^{\top} \widetilde{I} \bar{M}^{-1} \widetilde{I}^{\top} \mathbf{e}_{N}=\mathbf{e}_{N, \mathrm{R}}^{\top} M_{\mathrm{R}}^{-1} \mathbf{e}_{N, \mathrm{R}}$, we use the values tabulated in [3]. In Appendix B we show that (21) with (23) is stable.

\subsection{The Poisson equation}

We employ the method of manufactured solutions, and start by solving $-u_{x x}=f(x)$ with Dirichlet boundary conditions, using the steady version of the scheme (21) with $a=0$ and $\varepsilon=1$. In this case we use $\omega_{\mathrm{L}, \mathrm{R}}=q_{\mathrm{L}, \mathrm{R}}$ in (23). In the simulations, we are interested in the discrete $L^{2}$-norm of the solution error, i.e. $\|\mathrm{e}\|_{\tilde{P}}$, where $\mathbf{e}=\mathbf{u}-\underline{u}$ and $\|\mathrm{e}\|_{\widetilde{P}}^{2}=\mathrm{e}^{\top} \widetilde{P} \mathrm{e}$. 
We solve this problem with $f(x)=30^{2} \cos (30 x)$, such that the exact solution is $u=\cos (30 x)$, using schemes that changes order at $\hat{x}=0.5$. We construct one scheme using wide-stencil operators and one scheme using narrow-stencil operators, and let the left part have interior order 2 and the right part have interior order 6 . The resulting solutions and absolute values of the errors are shown in Figure 1, and the corresponding rates of convergence are given in Table 1. As expected, both schemes show second order convergence rate, which is the lowest order of accuracy of the included operators.
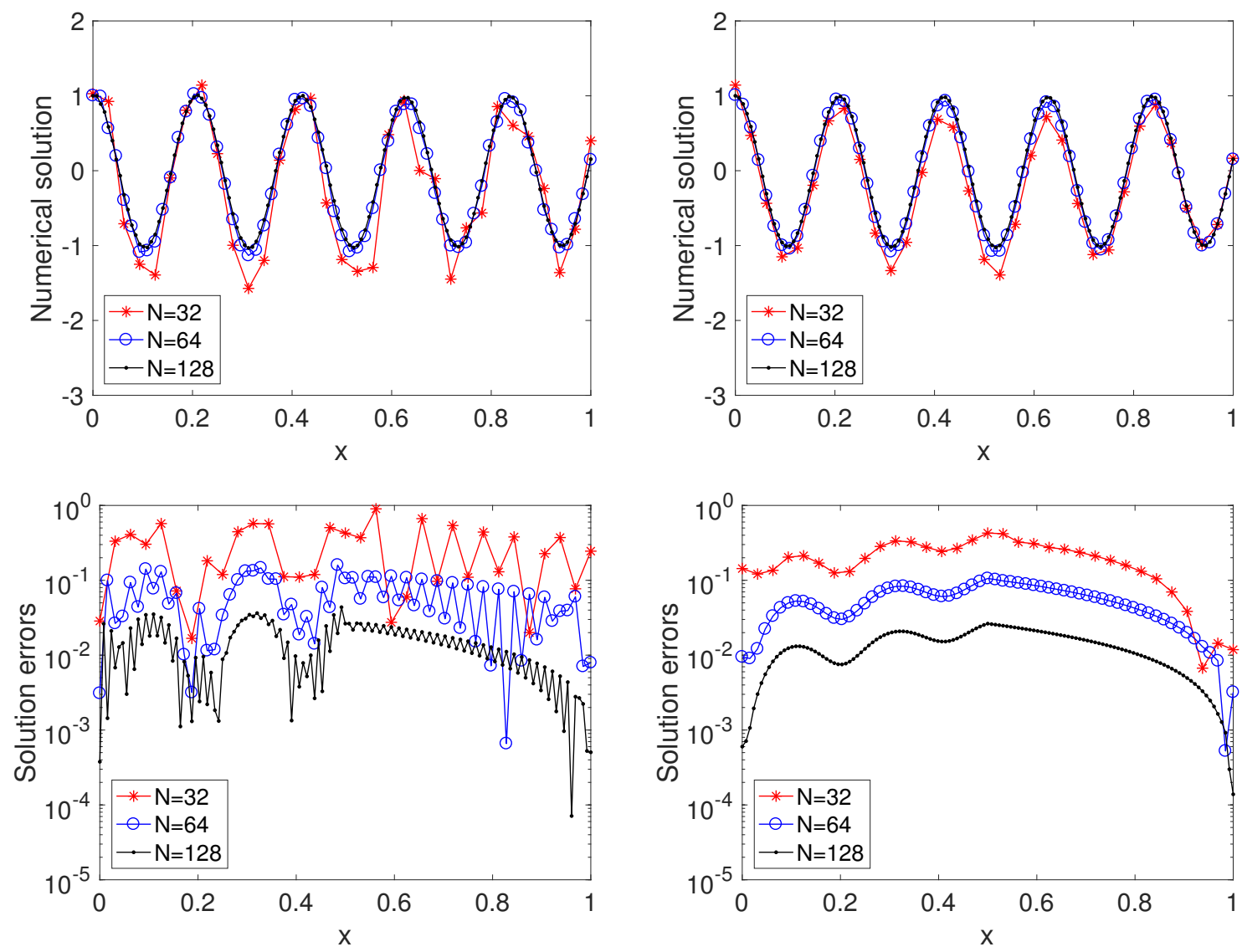

Figure 1: The schemes are composed by operators with interior order 2 to the left and interior order 6 to the right. The interface is positioned at $\hat{x}=0.5$ and the exact solution is $u=\cos (30 x)$. Left: Wide-stencil operators. Right: Narrow-stencil operators.

\begin{tabular}{|c|cc|cc|}
\hline & \multicolumn{2}{|c|}{ Wide-stencil operators } & \multicolumn{2}{c|}{ Narrow-stencil operators } \\
$\mathrm{N}$ & $\|\mathrm{e}\|_{\widetilde{P}}$ & $\log _{2}\left(\frac{\|\mathrm{e}(N / 2)\|_{\tilde{P}}}{\|\mathrm{e}(N)\|_{\tilde{P}}}\right)$ & $\|\mathrm{e}\|_{\widetilde{P}}$ & $\log _{2}\left(\frac{\|\mathrm{e}(N / 2)\|_{\tilde{P}}}{\|\mathrm{e}(N)\|_{\tilde{P}}}\right)$ \\
\hline 32 & 0.35539437 & - & 0.23478998 & - \\
64 & 0.07548625 & 2.2351 & 0.06015881 & 1.9645 \\
128 & 0.01786133 & 2.0794 & 0.01502129 & 2.0018 \\
256 & 0.00443970 & 2.0083 & 0.00375199 & 2.0013 \\
512 & 0.00110933 & 2.0008 & 0.00093777 & 2.0003 \\
1024 & 0.00027733 & 2.0000 & 0.00023443 & 2.0001 \\
\hline
\end{tabular}

Table 1: Errors and convergence rates corresponding to Figure 1. 


\subsubsection{Superconvergent functionals}

In many applications, functionals are more important than the primary solution itself (for example lift and drag coefficients in computational fluid dynamics). As mentioned, for operators $D_{1}$ with interior order of accuracy $2 p$ (which fulfill (4) with a diagonal $P$ ), the accuracy at the boundaries are restricted to order $p$ [9]. Similarly, narrow-stencil operators $D_{2}$ with interior order $2 p$ and boundary order $p$ (fulfilling (5) with a diagonal $P)$ are constructed in [11]. This, in turn, limits the order of accuracy of the resulting $L^{\infty}$-errors to $p+1$ for the wide-stencil operators and to $p+2$ for the narrow-stencil operators [14, 16]. For more details on accuracy, see also the discussions in $[18,17]$. Even so, when the scheme is dual consistent, the output functional can be computed with the full inner order of accuracy $2 p$ [8].

We consider linear functionals $\mathcal{J}(u)=\int_{\Omega} g u \mathrm{~d} x$, approximated by $J(\mathbf{u})=\mathbf{g}^{\top} \widetilde{P} \mathbf{u}$, and in addition to the solution error, we study the functional error $\mathrm{E}=|J(\mathbf{u})-\mathcal{J}(u)|$. The numerical simulations confirm that the new scheme preserves the superconvergence property. In fact, even when having a wide-stencil operator in one domain and a narrowstencil operator (with the same interior order) in the other domain, the functional converges with full order $2 p$. This is quite remarkable since the wide- and narrow-stencil operators produce solution errors of different orders. In Figure 2, the resulting errors when using schemes with interior order 6 are shown. The exact solution is $u=\cos (30 x)$ and the weight function is $g=\cos (30 x)$. Figure 2 also shows that the new scheme has the same global order of accuracy as the operator with the lowest order.
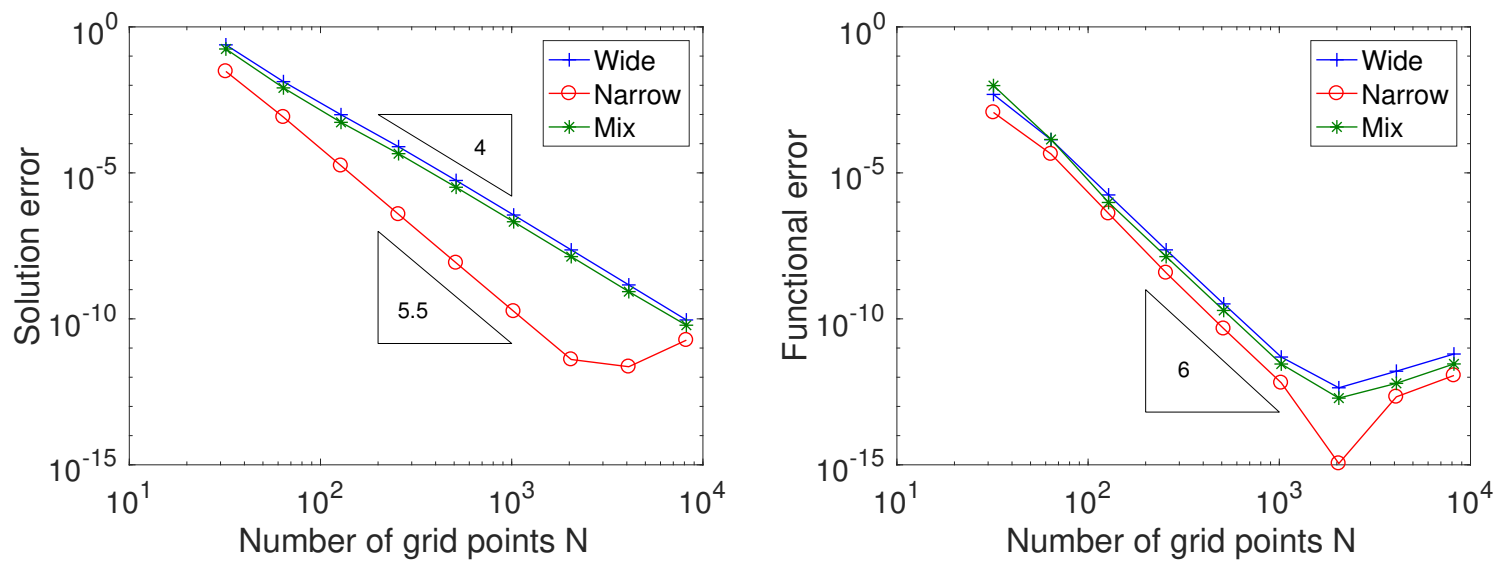

Figure 2: The mixed schemes are composed by wide-stencil operators to the left and narrowstencil operators to the right of the interface at $\hat{x}=0.5$. We use $u=g=\cos (30 x)$ and the operators have interior order 6. Left: Solution errors $\|\mathrm{e}\|_{\widetilde{P}}$. Right: Functional errors E.

\subsection{The advection-diffusion equation}

Next, we consider the steady version of (20). We use $a=1, \varepsilon=0.01, f=0, g_{\mathrm{L}}=1$ and $g_{\mathrm{R}}=0$, which leads to an exact solution with a steep gradient at $x=1$.

To solve this problem, we use the time-independent version of (21), with interior order 2 in the left domain (where the solution is almost flat), and interior order 6 in the right domain (where the boundary layer is located). The penalty parameters $\mu_{0}, \nu_{0}, \mu_{N}$ 
and $\nu_{N}$ are chosen according to $(23)$, with $\omega_{\mathrm{L}, \mathrm{R}}=|a|+\varepsilon q_{\mathrm{L}, \mathrm{R}}$ in case of narrow-stencil operators and $\omega_{\mathrm{L}, \mathrm{R}}=|a|$ in case of wide-stencil operators (this choice is derived to cancel oscillations [3]).

We first place the interface at $\hat{x}=0.5$, as before. The resulting numerical solutions and errors can be seen in Figure 3. The convergence plots that correspond to Figure 3 are shown in Figure 4 . Since the main contribution to $\|\mathrm{e}\|_{\widetilde{P}}$ stems from the right part of the domain, there is almost no difference between the new schemes with mixed order and the interface-free schemes with interior order 6 .
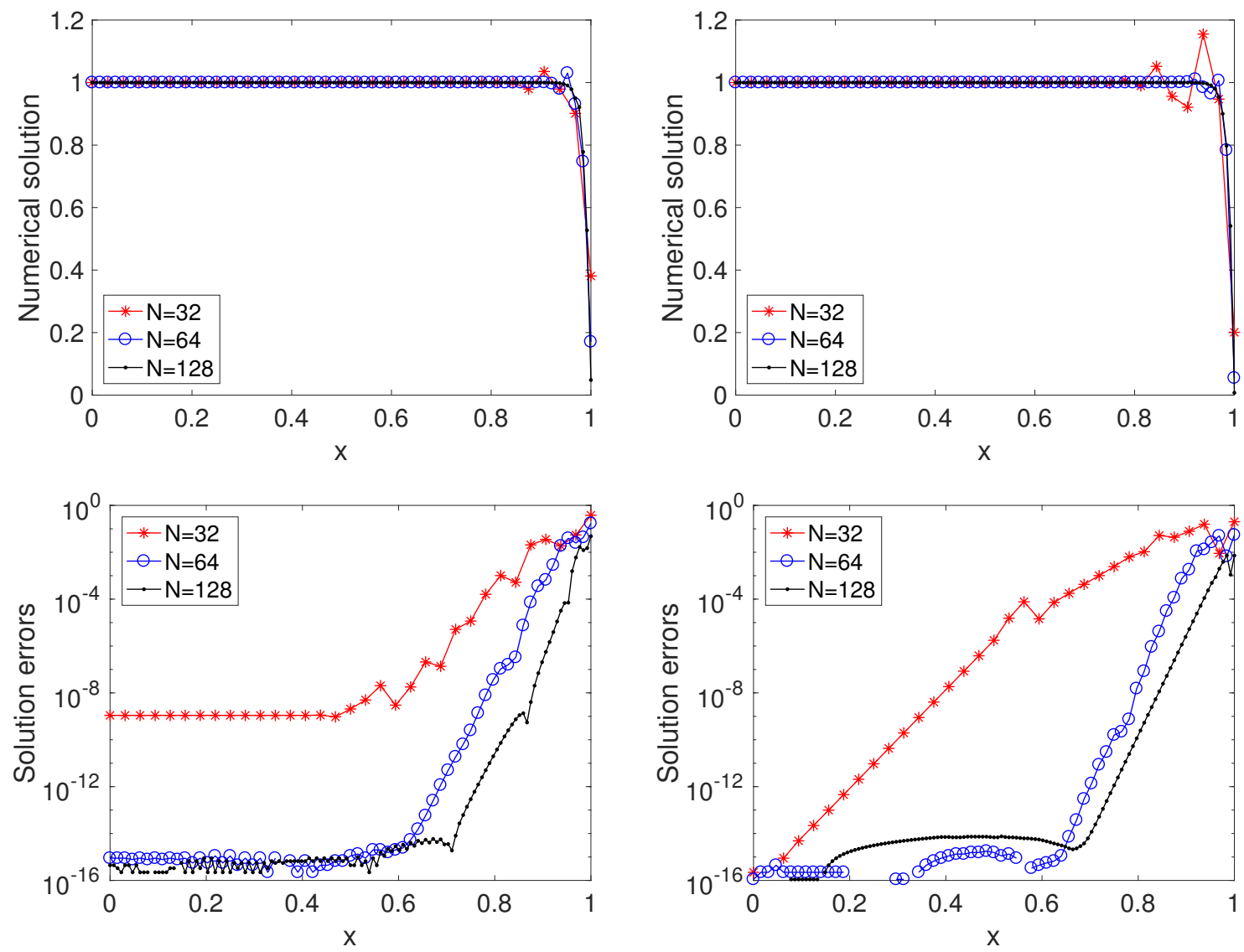

Figure 3: The schemes are composed by operators with interior order 2 in the left part of the domain and interior order 6 in the right part of the domain. The interface is positioned at $\hat{x}=0.5$. Left: Wide-stencil operators. Right: Narrow-stencil operators.

Remark 6.1. It is well known that wide-stencil schemes can produce oscillating solutions, but the oscillations decrease as the grid is refined [6]. However, thanks to the particular choice of penalty parameters used here, the interior oscillations are removed completely, as can be seen in Figure 3. In this case the wide-stencil schemes are actually preferable to the narrow-stencil schemes when the resolution is poor, i.e. when $|a| h / \varepsilon$ is large [3].

Finally, we place the interface at $\hat{x}=0.9375$ instead. (The standard SBP operators need at least $4 p$ grid points, and hence the new scheme under consideration needs $N_{\mathrm{R}} \geq 12$. We must thus use $N \geq 192$ when the interface is this close to the boundary.) The resulting convergence plots are displayed in Figure 5. Now the second order errors 
make an impact earlier, and we see that asymptotically, the new schemes converge with second order accuracy, as expected.
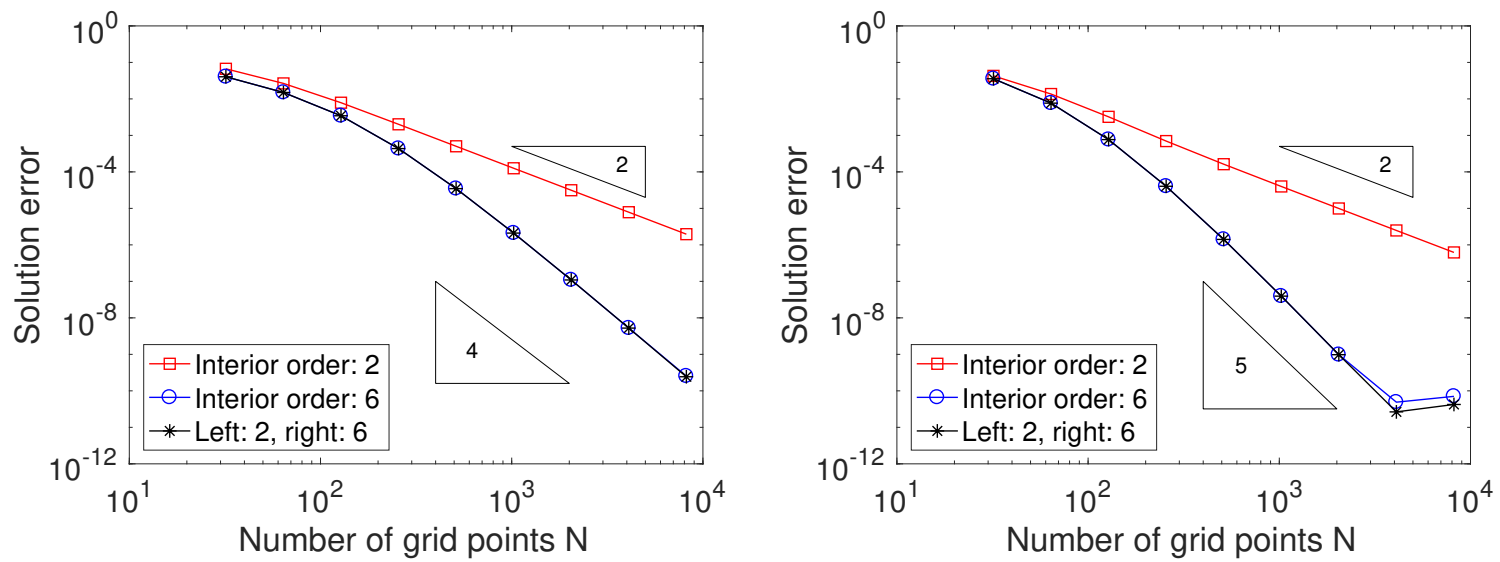

Figure 4: The convergence rates of $\|\mathrm{e}\|_{\widetilde{P}}$ corresponding to Figure 3 and compared with schemes without interfaces. Left: Wide-stencil operators. Right: Narrow-stencil operators.
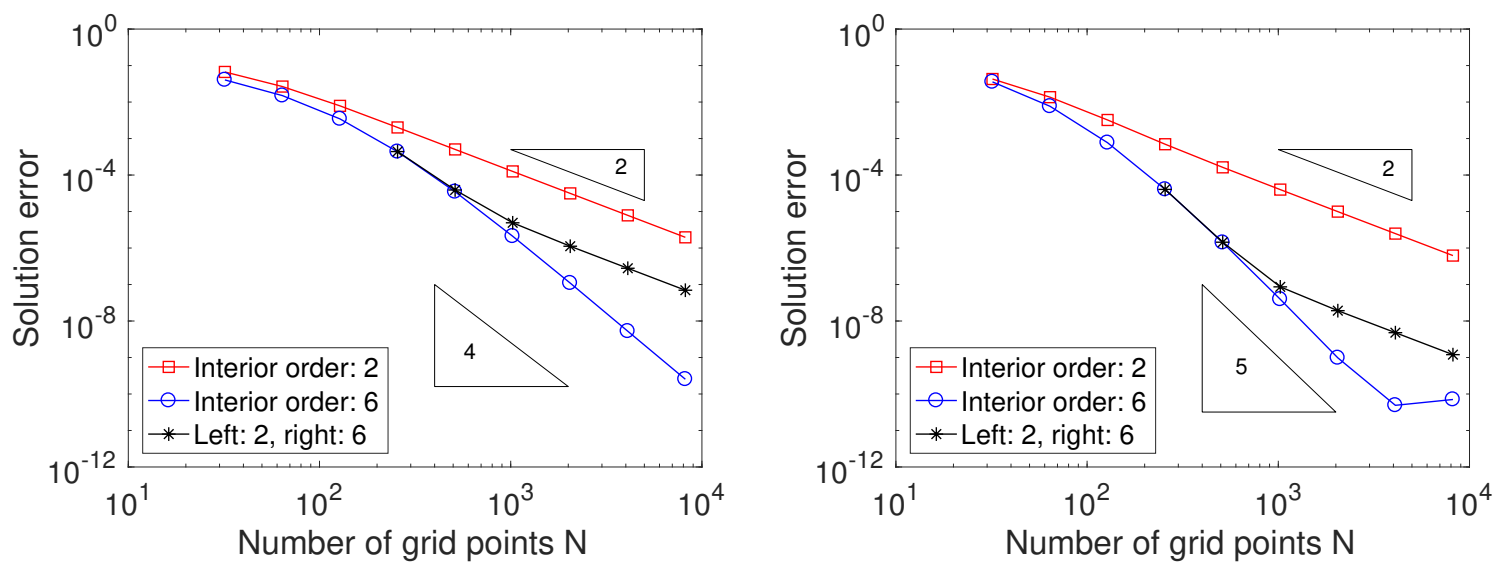

Figure 5: The convergence rates of $\|\mathrm{e}\|_{\widetilde{P}}$ when the interface is located in $\hat{x}=0.9375$. Left: Wide-stencil operators. Right: Narrow-stencil operators.

\section{Summary}

A procedure to locally change the type of finite difference stencils in a numerical scheme is developed. The development concerns operators approximating the second derivative, which complements work done earlier for the first derivative. The procedure is useful when the order of a numerical scheme has to be lowered or raised in a specific region of the computational domain.

The new operators are based on summation-by-parts operators with simultaneous approximation term interfaces, but modified such that the numerical solutions have a unique representation in all grid points. This facilitates the process of transferring the interface, which could be especially advantageous for time-dependent problems. 
The transition from one type of stencil to another is done in a time-stable and dual consistent manner, and the resulting operators have the same overall accuracy as the lowest included summation-by-parts operator. This is verified by numerical experiments on the Poisson equation and on the steady advection-diffusion equation.

\section{A Proofs of Proposition 4.1 and Corollary 4.3}

Proof of Proposition 4.1. Consider $\bar{P}$, which is given in (10), $\widetilde{K}$ and $\widetilde{I}$ from (16) and $\widetilde{P} \equiv \widetilde{I} \bar{P} \widetilde{I}^{\top}$. With $\alpha=\mathbf{e}_{N, \mathrm{~L}}^{\top} P_{\mathrm{L}} \mathbf{e}_{N, \mathrm{~L}} /\left(\mathbf{e}_{N, \mathrm{~L}}^{\top} P_{\mathrm{L}} \mathbf{e}_{N, \mathrm{~L}}+\mathbf{e}_{0, \mathrm{R}}^{\top} P_{\mathrm{R}} \mathbf{e}_{0, \mathrm{R}}\right)$ inserted into $\widetilde{K}$, we have

$$
\begin{aligned}
& \widetilde{P} \widetilde{K}=\left[\begin{array}{lllllllll}
\ddots & & & & & \\
& P_{\mathrm{L}}^{N_{\mathrm{L}}-1} & & & & \\
& & P_{\mathrm{L}}^{N_{\mathrm{L}}}+P_{\mathrm{R}}^{0} & & \\
& & & P_{\mathrm{R}}^{1} & \\
& & & & \ddots
\end{array}\right]\left[\begin{array}{cccccc}
\ddots & & & & & \\
& 1 & 0 & 0 & 0 & \\
& 0 & \frac{P_{\mathrm{L}}^{N_{\mathrm{L}}}}{P_{\mathrm{L}}^{N_{\mathrm{L}}}+P_{\mathrm{R}}^{0}} & \frac{P_{\mathrm{R}}^{0}}{P_{\mathrm{L}}^{N_{\mathrm{L}}}+P_{\mathrm{R}}^{0}} & 0 & \\
& 0 & 0 & 0 & 1 & \\
& & & & &
\end{array}\right] \\
& =\left[\begin{array}{cccccc}
\ddots & & & & & \\
& P_{\mathrm{L}}^{N_{\mathrm{L}}-1} & 0 & 0 & 0 & \\
& 0 & P_{\mathrm{L}}^{N_{\mathrm{L}}} & P_{\mathrm{R}}^{0} & 0 & \\
& 0 & 0 & 0 & P_{\mathrm{R}}^{1} & \\
& & & & & \ddots
\end{array}\right] \\
& =\left[\begin{array}{llllll}
\ddots & & & & & \\
& 1 & 0 & 0 & 0 & \\
& 0 & 1 & 1 & 0 & \\
& 0 & 0 & 0 & 1 & \\
& & & & & \ddots
\end{array}\right]\left[\begin{array}{lllllll}
\ddots & & & & & \\
& P_{\mathrm{L}}^{N_{\mathrm{L}}-1} & & & & \\
& & P_{\mathrm{L}}^{N_{\mathrm{L}}} & & & \\
& & & P_{\mathrm{R}}^{0} & & \\
& & & & P_{\mathrm{R}}^{1} & \\
& & & & & \ddots
\end{array}\right]=\widetilde{I} \bar{P},
\end{aligned}
$$

where $P_{\mathrm{L}}^{j}$ and $P_{\mathrm{R}}^{j}$ refer to the $j^{\text {th }}$ diagonal entry of $P_{\mathrm{L}}$ or $P_{\mathrm{R}}$, respectively. Having shown that $\widetilde{P} \widetilde{K}=\widetilde{I} \bar{P}$ for diagonal matrices $\bar{P}$, the relation $\widetilde{K} \bar{P}^{-1}=\widetilde{P}-1 \widetilde{I}$ follows.

Proof of Corollary 4.3. From the definitions of $\widetilde{K}$ and $\widetilde{I}$, given in (16), and $\overrightarrow{\mathbf{u}}$, which is given in (10), we compute

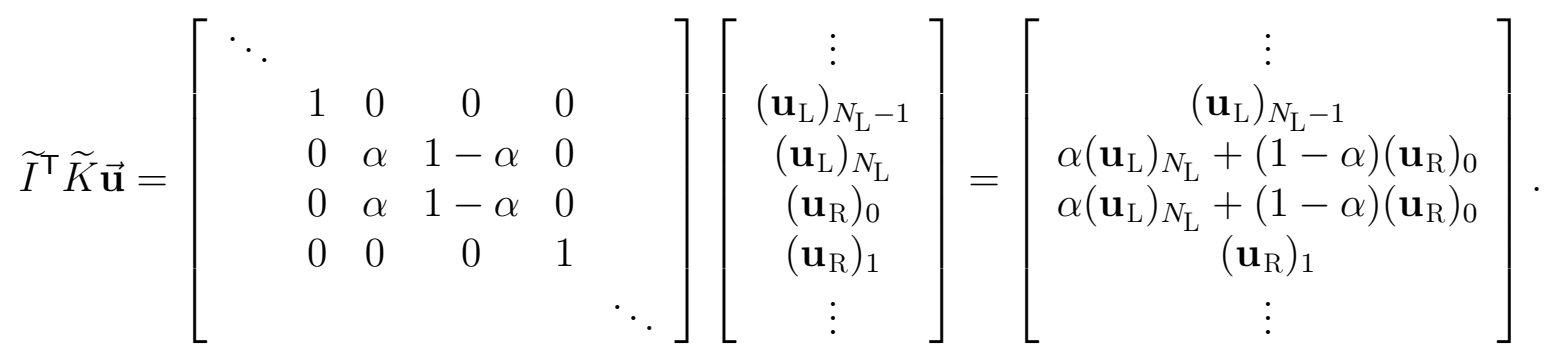

Assumption 4.2, that is that $\left(\mathbf{u}_{\mathrm{L}}\right)_{N_{\mathrm{L}}}=\left(\mathbf{u}_{\mathrm{R}}\right)_{0}$, leads to $\alpha\left(\mathbf{u}_{\mathrm{L}}\right)_{N_{\mathrm{L}}}+(1-\alpha)\left(\mathbf{u}_{\mathrm{R}}\right)_{0}=\left(\mathbf{u}_{\mathrm{L}}\right)_{N_{\mathrm{L}}}$ and $\alpha\left(\mathbf{u}_{\mathrm{L}}\right)_{N_{\mathrm{L}}}+(1-\alpha)\left(\mathbf{u}_{\mathrm{R}}\right)_{0}=\left(\mathbf{u}_{\mathrm{R}}\right)_{0}$. Thus $\overrightarrow{\mathbf{u}}=\widetilde{I}^{\top} \widetilde{K} \overrightarrow{\mathbf{u}}$ holds. 


\section{B Stability of the advection-diffusion scheme}

We multiply the scheme (21) (for simplicity with $\mathbf{f}=0$ and $g_{\mathrm{L}, \mathrm{R}}=0$ ) by $\mathbf{u}^{\top} \widetilde{P}$ from the left, and add the transpose. Thereafter using that $\widetilde{Q}+\widetilde{Q}^{\top}=\mathbf{e}_{N} \mathbf{e}_{N}^{\top}-\mathbf{e}_{0} \mathbf{e}_{0}^{\top}$, yields

$$
\begin{aligned}
\frac{\mathrm{d}}{\mathrm{d} t}\left(\mathbf{u}^{\top} \widetilde{P} \mathbf{u}\right)+2 \varepsilon \mathbf{u}^{\top} \widetilde{A} \mathbf{u} & =a \mathbf{u}^{\top} \mathbf{e}_{0} \mathbf{e}_{0}^{\top} \mathbf{u}-2 \varepsilon \mathbf{u}^{\top} \mathbf{e}_{0} \widetilde{\mathbf{d}}_{0}^{\top} \mathbf{u}+2 \mathbf{u}^{\top}\left(\mu_{0} \mathbf{e}_{0}+\nu_{0} \widetilde{\mathbf{d}}_{0}\right) \mathbf{e}_{0}^{\top} \mathbf{u} \\
& -a \mathbf{u}^{\top} \mathbf{e}_{N} \mathbf{e}_{N}^{\top} \mathbf{u}+2 \varepsilon \mathbf{u}^{\top} \mathbf{e}_{N} \widetilde{\mathbf{d}}_{N}^{\top} \mathbf{u}+2 \mathbf{u}^{\top}\left(\mu_{N} \mathbf{e}_{N}+\nu_{N} \widetilde{\mathbf{d}_{N}}\right) \mathbf{e}_{N}^{\top} \mathbf{u} .
\end{aligned}
$$

Next, we define $\widetilde{\mathbf{w}}=\bar{S} \widetilde{I}^{\top} \mathbf{u}+\bar{M}^{-1} \widetilde{I}^{\top} \mathbf{e}_{0} \mathbf{e}_{0}^{\top} \mathbf{u}-\bar{M}^{-1} \widetilde{I}^{\top} \mathbf{e}_{N} \mathbf{e}_{N}^{\top} \mathbf{u}$, with $\bar{S}$ and $\bar{M}$ given in (22), and compute

$$
\widetilde{\mathbf{w}}^{\top} \bar{M} \widetilde{\mathbf{w}}=\mathbf{u}^{\top} \widetilde{A} \mathbf{u}+2 \widetilde{\mathbf{d}}_{0}^{\top} \mathbf{u} \mathbf{e}_{0}^{\top} \mathbf{u}-2 \widetilde{\mathbf{d}}_{N}^{\top} \mathbf{u} \mathbf{e}_{N}^{\top} \mathbf{u}+q_{\mathrm{L}}\left(\mathbf{e}_{0}^{\top} \mathbf{u}\right)^{2}+q_{\mathrm{R}}\left(\mathbf{e}_{N}^{\top} \mathbf{u}\right)^{2},
$$

where we have exploited that

$$
\begin{aligned}
\mathbf{e}_{0}^{\top} \widetilde{I} \bar{M}^{-1} \widetilde{I}^{\top} \mathbf{e}_{0}=\mathbf{e}_{0, \mathrm{~L}}^{\top} M_{\mathrm{L}}^{-1} \mathbf{e}_{0, \mathrm{~L}} \equiv q_{\mathrm{L}}, & \mathbf{e}_{N}^{\top} \widetilde{I} \bar{M}^{-1} \widetilde{I}^{\top} \mathbf{e}_{0}=0, \\
\mathbf{e}_{N}^{\top} \widetilde{I} \bar{M}^{-1} \widetilde{I}^{\top} \mathbf{e}_{N}=\mathbf{e}_{N, \mathrm{R}}^{\top} M_{\mathrm{R}}^{-1} \mathbf{e}_{N, \mathrm{R}} \equiv q_{\mathrm{R}}, & \mathbf{e}_{0}^{\top} \widetilde{I} \bar{M}^{-1} \widetilde{I}^{\top} \mathbf{e}_{N}=0 .
\end{aligned}
$$

Using (25) in (24) leads to the energy growth rate

$$
\frac{\mathrm{d}}{\mathrm{d} t}\left(\mathbf{u}^{\top} \widetilde{P} \mathbf{u}\right)+2 \varepsilon \widetilde{\mathbf{w}}^{\top} \bar{M} \widetilde{\mathbf{w}}=-\omega_{\mathrm{L}}\left(\mathbf{e}_{0}^{\top} \mathbf{u}\right)^{2}-\omega_{\mathrm{R}}\left(\mathbf{e}_{N}^{\top} \mathbf{u}\right)^{2} \leq 0,
$$

where we have also used the choice of penalty parameters suggested in (23).

Remark B.1. In contrast to what is generally the case when using narrow-stencil second derivative operators, the modification of $q_{\mathrm{L}, \mathrm{R}}$ mentioned in Remark 3.3 is not needed. This is because $\bar{M}$ is block-diagonal, which leads to $\mathbf{e}_{N}^{\top} \widetilde{I} \bar{M}^{-1} \widetilde{I}^{\top} \mathbf{e}_{0}=\mathbf{e}_{0}^{\top} \widetilde{I} \bar{M}^{-1} \widetilde{I}^{\top} \mathbf{e}_{N}=0$.

\section{References}

[1] J. Berg and J. Nordström. On the impact of boundary conditions on dual consistent finite difference discretizations. Journal of Computational Physics, 236:41-55, 2013 .

[2] M. H Carpenter, J. Nordström, and D. Gottlieb. A stable and conservative interface treatment of arbitrary spatial accuracy. Journal of Computational Physics, 148(2):341-365, 1999.

[3] S. Eriksson. A dual consistent finite difference method with narrow stencil second derivative operators. Journal of Scientific Computing, DOI: 10.1007/s10915-0170569-6, 2017.

[4] S. Eriksson, Q. Abbas, and J. Nordström. A stable and conservative method for locally adapting the design order of finite difference schemes. Journal of Computational Physics, 230(11):4216-4231, 2011. 
[5] D. C. Del Rey Fernández, J. E. Hicken, and D. W. Zingg. Review of summation-byparts operators with simultaneous approximation terms for the numerical solution of partial differential equations. Computers \& Fluids, 95:171-196, 2014.

[6] H. Frenander and J. Nordström. Spurious solutions for the advection-diffusion equation using wide stencils for approximating the second derivative. Numerical Methods for Partial Differential Equations, 34(2):501-517, 2018.

[7] B. Gustafsson, H.-O. Kreiss, and J. Oliger. Time-Dependent Problems and Difference Methods. John Wiley \& Sons, Inc., 2013.

[8] J. E. Hicken and D. W. Zingg. Superconvergent functional estimates from summation-by-parts finite-difference discretizations. SIAM Journal on Scientific Computing, 33(2):893-922, 2011.

[9] H.-O. Kreiss and G. Scherer. Finite element and finite difference methods for hyperbolic partial differential equations, in: Mathematical Aspects of Finite Elements in Partial Differential Equations. Academic Press, Inc., 1974.

[10] V. Linders, T. Lundquist, and J. Nordström. On the order of accuracy of finite difference operators on diagonal norm based summation-by-parts form. Accepted in SIAM Journal on Numerical Analysis, 2018.

[11] K. Mattsson and J. Nordström. Summation by parts operators for finite difference approximations of second derivatives. Journal of Computational Physics, 199(2):503-540, 2004.

[12] J. Nordström, J. Gong, E. van der Weide, and M. Svärd. A stable and conservative high order multi-block method for the compressible Navier-Stokes equations. Journal of Computational Physics, 228(24):9020-9035, 2009.

[13] J. Nordström and M. Svärd. Well-posed boundary conditions for the Navier-Stokes equations. SIAM Journal on Numerical Analysis, 43(3):1231-1255, 2005.

[14] M. Svärd and J. Nordström. On the order of accuracy for difference approximations of initial-boundary value problems. Journal of Computational Physics, 218(1):333$352,2006$.

[15] M. Svärd and J. Nordström. Review of summation-by-parts schemes for initialboundary-value problems. Journal of Computational Physics, 268:17-38, 2014.

[16] M. Svärd and J. Nordström. On the convergence rates of energy-stable finitedifference schemes. Technical Report LiTH-MAT-R-2017/14-SE, Linköping University, Department of Mathematics, 2017.

[17] M. Svärd and J. Nordström. Response to "Convergence of summation-by-parts finite difference methods for the wave equation". Journal of Scientific Computing, 74(2):1188-1192, 2018.

[18] S. Wang and G. Kreiss. Convergence of summation-by-parts finite difference methods for the wave equation. Journal of Scientific Computing, 71(1):219-245, 2017. 